\title{
1. Dynamic Boolean modelling reveals the influence of energy supply on bacterial efflux pump expression
}

\author{
Ryan Kerr ${ }^{1,2}$, Sara Jabbari ${ }^{1,2, *}$, Jessica M. A. Blair², and lain G. Johnston ${ }^{3,4, *}$ \\ ${ }^{1}$ School of Mathematics, University of Birmingham, United Kingdom \\ ${ }^{2}$ Institute of Microbiology and Infection, University of Birmingham, United Kingdom \\ ${ }^{3}$ Department of Mathematics, Faculty of Mathematics and Natural Sciences, University of Bergen, Bergen, Norway \\ ${ }^{4}$ Computational Biology Unit, University of Bergen, Norway \\ *Correspondence to s.jabbari@bham.ac.uk (SJ) or iain.johnston@uib.no (IGJ)
}

\begin{abstract}
Antimicrobial resistance (AMR) is a global health issue. One key factor contributing to AMR is the ability of bacteria to export drugs through efflux pumps, which relies on the ATP-dependent expression and interaction of several controlling genes. Recent studies have shown significant cell-to-cell ATP variability exists within clonal bacterial populations, but the contribution of intrinsic cell-to-cell ATP heterogeneity is generally overlooked in understanding efflux pumps. Here, we consider how ATP variability influences gene regulatory networks controlling expression of efflux pump genes in two bacterial species. We develop and apply a generalisable Boolean modelling framework, developed to incorporate the dependence of gene expression dynamics on available cellular energy supply. Theoretical results show differences in energy availability can cause pronounced downstream heterogeneity in efflux gene expression. Cells with higher energy availability have a superior response to stressors. Further, in the absence of stress, model bacteria develop heterogeneous pulses of efflux pump gene expression which contribute to a sustained sub-population of cells with increased efflux expression activity, potentially conferring a continuous pool of intrinsically resistant bacteria. This modelling approach thus reveals an important source of heterogeneity in cell responses to antimicrobials and sheds light on potentially targetable aspects of efflux pump-related antimicrobial resistance.
\end{abstract}

${ }_{25}$ Keywords

26 ATP

27 efflux pumps

${ }_{28} \quad$ acrAB

29 Boolean modelling

so cell-to-cell variability

${ }_{31}$ heterogeneous pulsing 


\section{Introduction}

Antimicrobial resistance (AMR) is a huge global health challenge, contributing to over 700,000 deaths worldwide [1] and estimated to exceed cancer mortality by 2050 if neglected [2]. Protein complexes called efflux pumps are an important mechanism for intrinsic (and acquired) resistance to antibiotics [3-5]. Efflux pumps are capable of transporting a range of substances from the cell compartment to the extracellular medium, reducing intracellular accumulation of substances including antibiotics, and thus conferring resistance. Their central role in facilitating clinically relevant AMR makes efflux pumps an attractive drug target, with much research directed at efflux pump inhibitors as a novel therapeutic to overcome efflux-mediated resistance [6-10].

A current mystery in the activity of efflux pumps is the causes and effects of cell-to-cell heterogeneity in their expression. Recent data demonstrates efflux pump gene expression is not homogeneous throughout the population - instead, bacterial populations display significant cell-to-cell variability [11-13]. Heterogeneity in efflux activity has been suggested to have further downstream influence on AMR. One study, by Sánchez-Romero and Casadesús [13], reported a correlation between bacteria with high efflux pump activity in the absence of antibiotic compounds and increased resistance to antibiotics. Additionally, a study by El Meouche and Dunlop [11] has shown a sub-population of bacteria with higher efflux pump expression has lower expression of mismatch repair genes, facilitating mutations which can lead to permanent genetic changes bestowing resistance. However, the underlying dynamics and influences of efflux heterogeneity in cellular populations remain to be uncovered.

One potential source of heterogeneity in gene expression dynamics and interactions is cell-to-cell differences in available energy supply $[14,15]$. Heterogeneity in adenosine triphosphate (ATP) levels in bacteria (and other species; see Discussion) is increasingly being characterised experimentally [16,17]. The quantified range of intracellular ATP can cover almost an order of magnitude, varying between $0.32-2.76 \mathrm{mM}$ in Escherichia coli [16], for example. Although not uncontroversial, the ubiquity of cell-to-cell variability in ATP across life suggests it is a genuine effect [18-20]. Following theoretical work showing the potentially profound influence of this variability on gene regulatory networks $[14,15]$, we here investigate the hypothesis that cell-to-cell differences in available energy can contribute to cell heterogeneity in efflux pump expression, intrinsic resistance to antimicrobials, and response to different environmental stressors.

We previously applied an energy-dependent ordinary differential equation (ODE) framework to a simple GRN, describing the behaviour of many naturally-occurring decision-making circuits [14]. We predicted that differences in cellular energy levels will cause differences in the dynamics and stable outcomes of cellular decision-making. However, while ODE models can in principle be used for GRNs of arbitrary size, the associated parameter space rapidly increases with network size, making it harder to investigate general principles. Here, we develop and use an alternative theoretical framework based on Boolean network models [21,22], simplifications that have been successfully applied to diverse biological systems [23-25] (although to date not often used to understand bacterial virulence and resistance mechanisms [26,27]). Despite this widespread and successful use, there has been little consideration of the fact that the processes in Boolean GRN models correspond biologically to ATP-dependent processes $[21,26,28]$. This ATP dependence is important, because it generally influences the dynamics of gene expression [29]. We therefore proceed by developing a simple but highly generalisable modification for including energy dependence in Boolean models of regulatory networks, and use this theoretical framework to explore the effects of ATP variability in experimentally-derived GRN models of efflux pump expression in E. coli and Salmonella.

\section{Results}

\subsection{Literature-based model construction accounting for energy availability}

We first sought to construct a Boolean modelling framework describing efflux pump expression dynamics, supported by data and yielding verifiable predictions about biological behaviour. We considered AcrABTolC, an efflux pump associated with clinically important drug resistance in E. coli [30-32] and Salmonella 
(A)

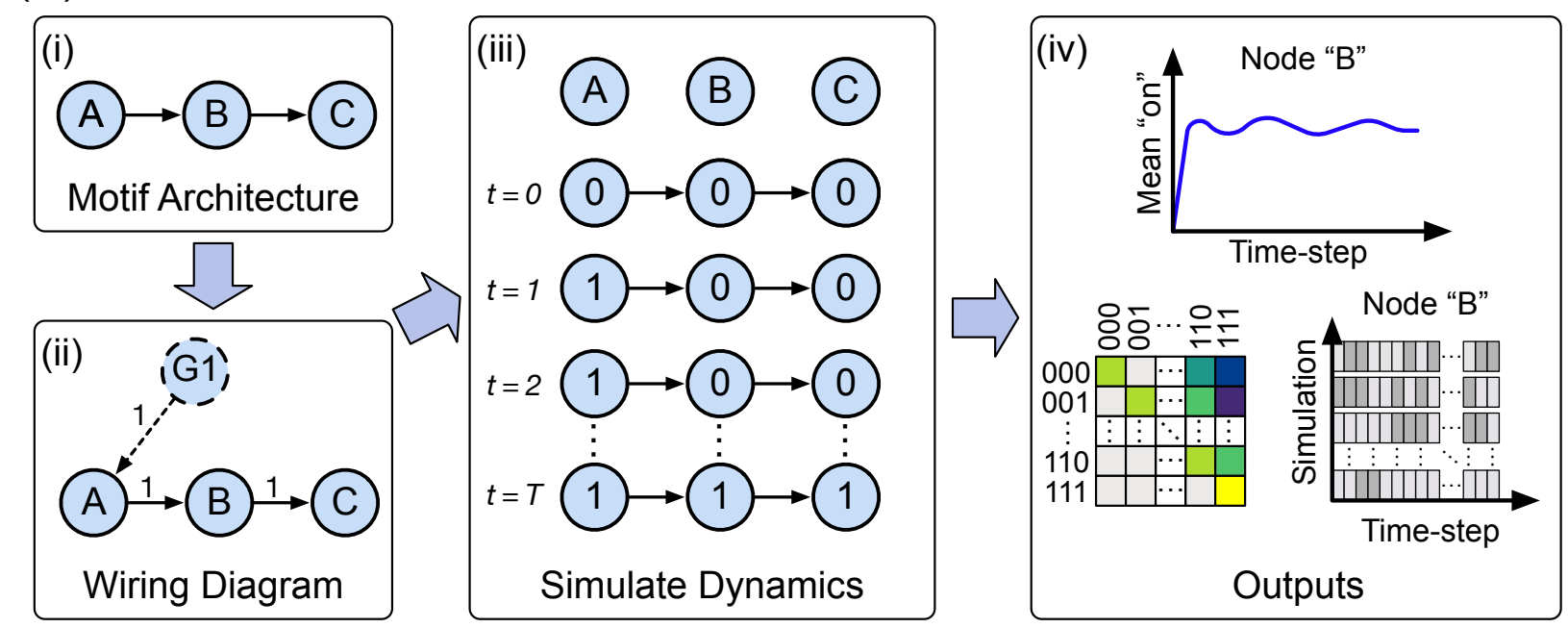

(B)

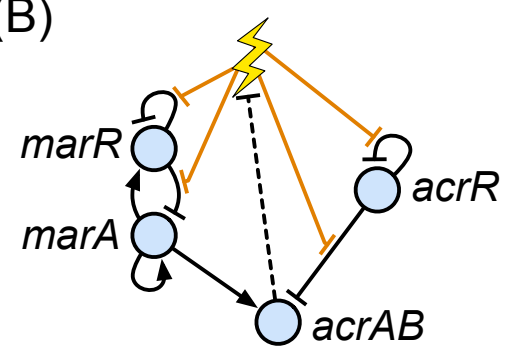

(C)

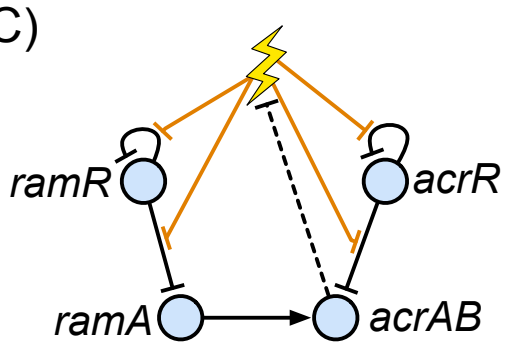

Figure 1: Boolean network modelling of gene regulatory network architectures controlling efflux pump gene expression. (A) Schematic overview of the Boolean network framework. Regulatory architectures (i) are converted into wiring diagram form (ii). These wiring diagrams include 'ghost nodes' (G1) if necessary, providing a constant signal to ensure constitutively expressed genes are "on" unless switched "off" by a regulator. The considered network is repeatedly simulated over a set number of timesteps (iii), from some initial configuration, producing individual and averaged pictures of network behaviour through transition matrices and expression dynamics of each node per timestep (iv). (B-C) Coarse-grained E. coli (B) and Salmonella (C) AcrAB efflux pump gene regulatory networks, containing a collection of genetic regulators (blue circles) that interact with each other (directed edges) and govern the expression level of efflux genes acrAB. Full layouts with parameterizations are given in Supplementary Fig. S1. Antibiotics, or other noxious stressor substances (lightning bolt), can reversibly bind to a gene product (transcription factor) removing its ability to bind to DNA and regulate its target (displayed through orange edges), with consequent downstream effects through the network. Removal of the stressor is integrated through a repressive interaction from acr $A B$ to the stress node (dotted edges). 
[33-36]. ToIC is constitutively expressed [37] and predicted to be integrated within at least seven additional efflux transport systems in $E$. coli [38], suggesting the dynamics of acr $A B$ are more limiting on producing the complete efflux pump system within each cell. We performed a broad literature review (see Supplementary Information Section 5.1) of efflux pump gene regulation in E. coli and Salmonella to define a core GRN of $a c r A B$ in each species (Fig. 1(B)-(C)). This review defined the structure of our model i.e. the nodes, regulatory interactions and edge weights in the corresponding wiring diagrams (Supplementary Fig. S1(A)(B)).

E. coli contains a global activator of efflux genes acrAB, called marA (of the marRAB operon), which is repressed locally by the product of marR [39]. Similarly, in Salmonella, a global activator $\operatorname{ram} A$ is repressed locally by RamR, the product of ramR [40]. In both networks, repressor AcrR, the gene product of acrR, down-regulates its own expression and prevents over-expression of efflux genes acrAB [41] (see Fig. 11(B)(C) for each complete GRN). acrAB encodes the proteins AcrA and AcrB [42], which can assemble together with TolC to form the AcrAB-TolC system $[43,44]$ that provides the physical means to expel unwanted substances from the cell (such as toxic metabolic intermediates or bile salts $[45,46]$ ).

The expression of efflux pumps is inducible by the presence of environmental signals, or chemical compounds (such as antibiotics), which we will generally refer to as 'stressors'. The inducibility of efflux genes enables bacteria to adapt to a wide range of environmental conditions. Noxious substances, such as antibiotics, can bind to GRN products MarR, RamR and AcrR, inhibiting their ability to bind to DNA, and initiating a response cascade that leads to the up-regulation of efflux genes acrAB [47-52]. In our model the expression of acr $A B$ corresponds to the capacity of the assembled efflux pump to export molecules.

To avoid the aforementioned parameterization issues in continuous models, we developed a general Boolean framework that captures the influence of energy availability. Our model represents genes as nodes in a network, where each gene can be "on" (expressed) or "off" (not expressed) at a given time. Edges describe activating and repressing interactions, and the state of a network changes over time with asynchronous update rules following these interactions (see Methods), capturing the stochastic nature of gene regulatory dynamics. To explicitly capture the fact that the fundamental processes (hence all gene interaction "arrows") involved in gene expression depend on ATP, we modulate the rate with which regulatory interactions from node $X$ to node $Y$ are applied according to cellular energy levels (see Methods). This reflects the fact that, for example, cells with low ATP will have a lower rate of transcription elongation per timestep [53], slowing the dynamics of the biochemical intermediates involved in these regulatory interactions, and thus slowing the interactions themselves [14]. While fast fluctuations in ATP supply may exist within cells, as gene expression processes are dynamically slower, we assume the network is exposed to a time-averaged ATP level, and thus define three energy levels 'high', 'intermediate', and 'low', respectively involving a characteristic mean rate of $1,1 / 2$, and $1 / 10$ per simulation, corresponding to the aforementioned order of magnitude range observed in biology (see Methods).

\subsection{Efflux network models reproduce experimentally observed behaviour in E. coli and Salmonella}

Having constructed our model based on a set of experimental observations, we next asked whether it made predictions that could be tested using independent experimental data. In the Supplementary Information we present a table assessing the predictions of our model, for our choice of edge weights, with respect to a multiscale set of experiments at both the population and single-cell level, where agreement is demonstrated across a wide variety of observations (Supplementary Table S1. The overall behaviour of both systems - which we will describe in detail below - predicts a range of coarse- and fine-grained features observed in independent experiments, including pronounced gene expression heterogeneity at the single-cell level, prominent increases in gene expression when a short or long stress signal is provided, the expression of repressive genes in response to stress [41,54]), and the details of expression levels of different actors in the GRN in the presence and absence of stress. 
(i)

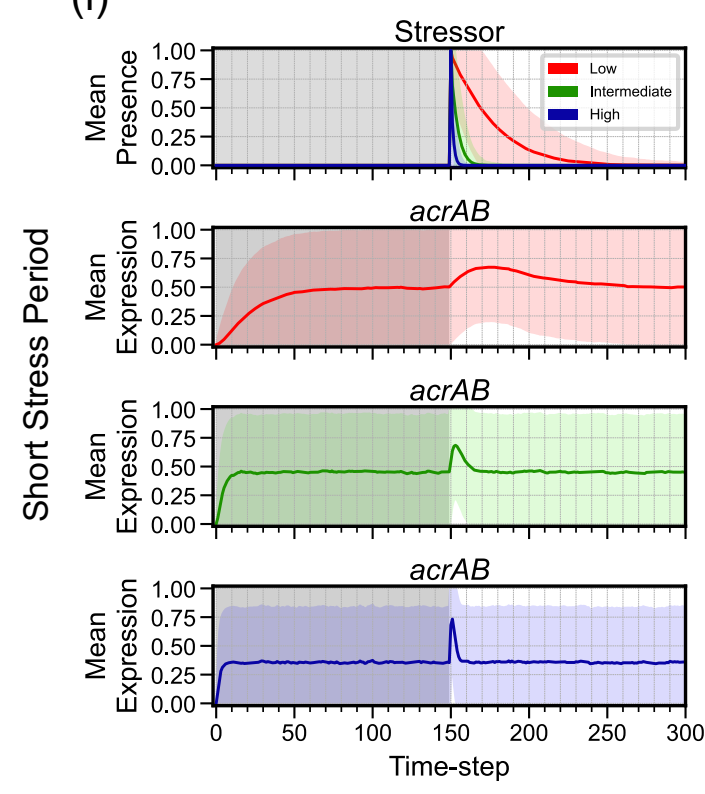

(iii)

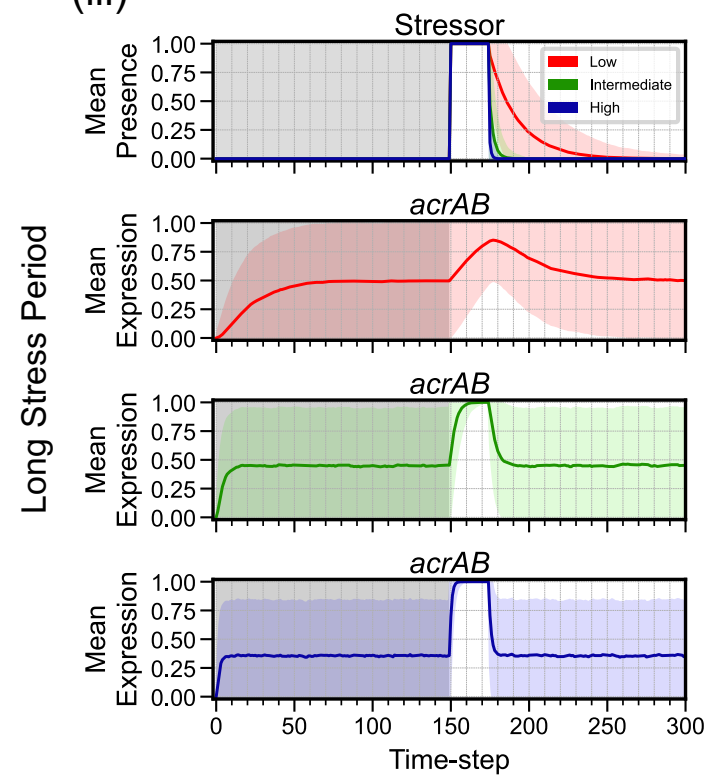

(ii)
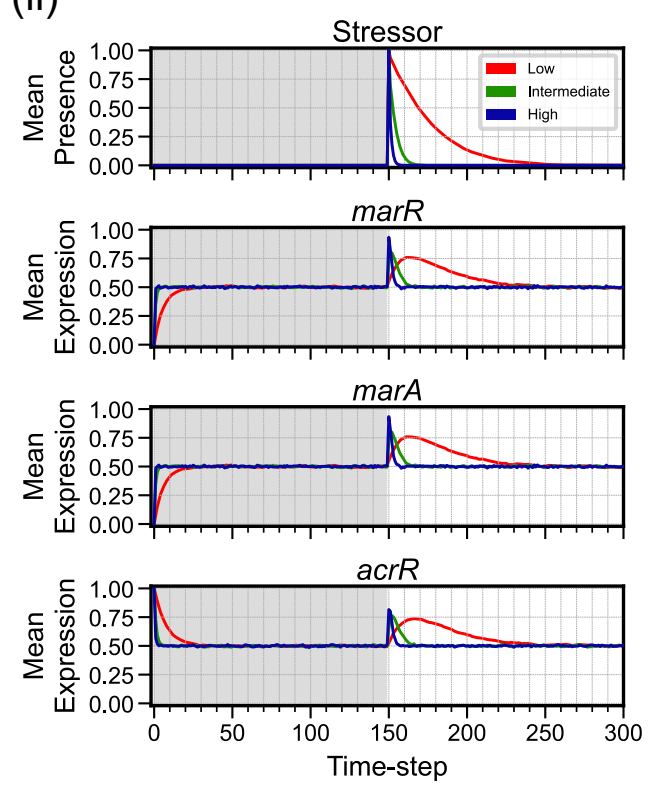

(iv)
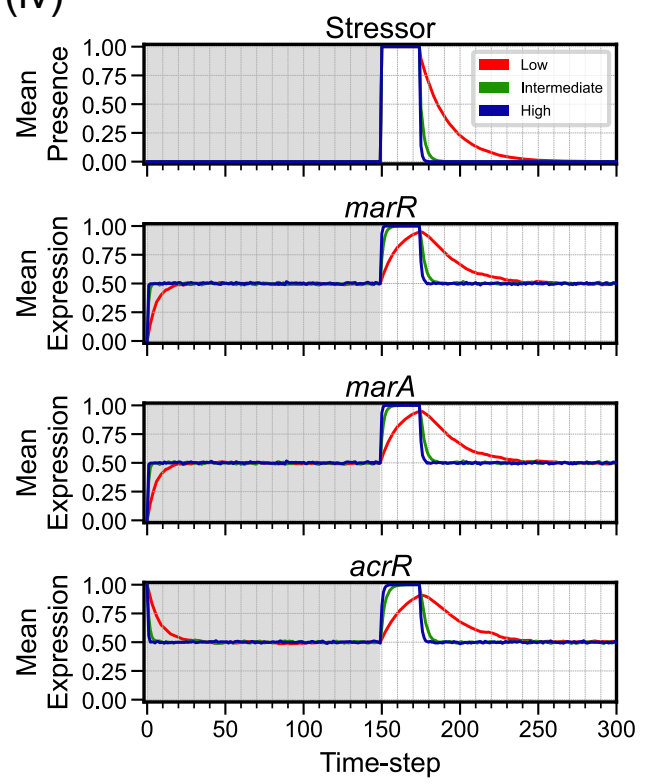

Figure 2: Mean expression dynamics for $E$. coli from the unstressed initial condition in response to a short or long stress period. Time-series dynamics of mean expression (coloured line for low (red), intermediate (green) and high (blue) energy levels) for ((i), (iii)) efflux genes $\operatorname{acr} A B$ and ((ii), (iv)) remaining regulatory components in $E$. coli; all panels include stressor behaviour. Behaviour is shown when the network is exposed to a (i)-(ii) short $(t=150)$ or (iii)-(iv) long $(t=150-174)$ stress period, from the unstressed initial condition. The initial time region of each regulatory component prior to stress application (grey shaded region) displays the complete stress-free behaviour. Note: Standard deviation (corresponding coloured shaded area) is included within efflux panels to represent the uncertainty, but the actual expression level cannot exceed the $[0,1]$ domain. 


\subsection{Stress-free efflux network behaviour with a maximal cellular energy budget}

To dissect the dynamics of these networks in detail, we first explored the stress-free population-level behaviour of each network. In the absence of stress, the population-wide mean behaviour in expression levels approaches a steady state (pre-stress dynamics in Fig. 2). We found at high energy availability, initial conditions had no influence on this population-level equilibrium of the E. coli and Salmonella networks (see Supplementary Information Section 5.5. We therefore proceed by investigating network behaviour starting from a particular initial state - which models an 'unstressed' baseline condition - in the rest of the study and focus on the $E$. coli network before comparing the behaviour of the two networks.

To explore the variability within the equilibrium population without energetic limitations, we calculated the expression level coefficient of variation (standard deviation divided by mean) for each genetic component in the E. coli network. In the absence of stress, expression level coefficient of variation (ELCV), corresponding to population variability in gene expression, ranged between 1.0-1.35 at equilibrium (see stress-free period in Fig. 2). This diversity is due to population-level asynchrony in expression dynamics; in simulated individual bacteria, network components are expressed in heterogeneous pulses (pre-stress dynamics in Fig. 3 . This diversity, particularly in $a c r A B$, suggests that the network structure itself may support a degree of 'bet-hedging' in the absence of stress, where some members of a population are primed to respond to stressors at any given time, agreeing with experimentally observed behaviour in Salmonella enterica [13] (see Discussion).

\subsection{Efflux network behaviour with a maximal cellular energy budget after exposure to a stressor}

We next asked how exposure to a stressor affects network behaviour. Following a simulated stress-free period as in the previous section, we simulated exposure to a stressor for either a short (one timestep) or long (25 timesteps) stress period. In response to stress, the mean expression levels of all genes in the system change, with different rates and magnitude (Fig. 2). acrAB mean expression rises throughout stress exposure, thus becoming higher for the longer stress duration (Fig. 2(i) and (iii)). After the long stress period, the high energy population displays homogeneous behaviour (active efflux expression), increasing the capacity of each cell to remove the stressor (see Fig. 3 (iv) to qualitatively observe this behaviour). The high energy population can then rapidly, and collectively, expel the noxious substance, promptly restoring baseline conditions in mean expression following the steps discussed previously.

When stimulated by a short stress (Fig. 22(i)), high variability is observed in efflux pump activity throughout the entire stress response, corresponding to diverse responses across the bacterial population. Individual simulation dynamics (Fig. 3 (i)) suggest this behaviour originates from sub-populations of cells developing unsynchronised responses to stress, and a delayed development of the efflux response. The unsynchronised dynamics prolong the population-level stress response, potentially benefiting a clonal population by providing a hedging mechanism against future stress.

Once the simulated stressor is removed, repressive transcription factors resume down-regulation of their GRN target, initially reducing the mean expression level of marRA and acrR, followed by a reduction in acr $A B$ mean expression (Fig. 2). This process switches off the response cascade, allowing acr $A B$ mean expression to return to the pre-stress population equilibrium (Fig. 2).

We next asked when the population of $E$. coli cells reached an equilibrium in acr $A B$ expression after each perturbation - that is, when the statistics of efflux gene expression levels no longer changed with time in the population after the stress period. We found that the number of timesteps for acrAB to attain a poststress population equilibrium was similar after a short or long stress ( 9 timesteps vs 8 timesteps respectively, Fig. 2 (i) and (iii)), and the equilibrium eventually attained was the same for both stress durations.

To understand these dynamics in more depth, we next asked how the different patterns of gene expression between which a network can transition vary when a cell is exposed to stress (reflecting the diversity of behaviours supported by the network). We define 'accessible transitions' to be the set of expression patterns that can be reached directly from a given network state. To explore this, we analysed the transition 
bioRxiv preprint doi: https://doi.org/10.1101/2021.12.13.472361; this version posted December 14, 2021. The copyright holder for this preprint (which was not certified by peer review) is the author/funder, who has granted bioRxiv a license to display the preprint in perpetuity. It is made available under aCC-BY 4.0 International license.
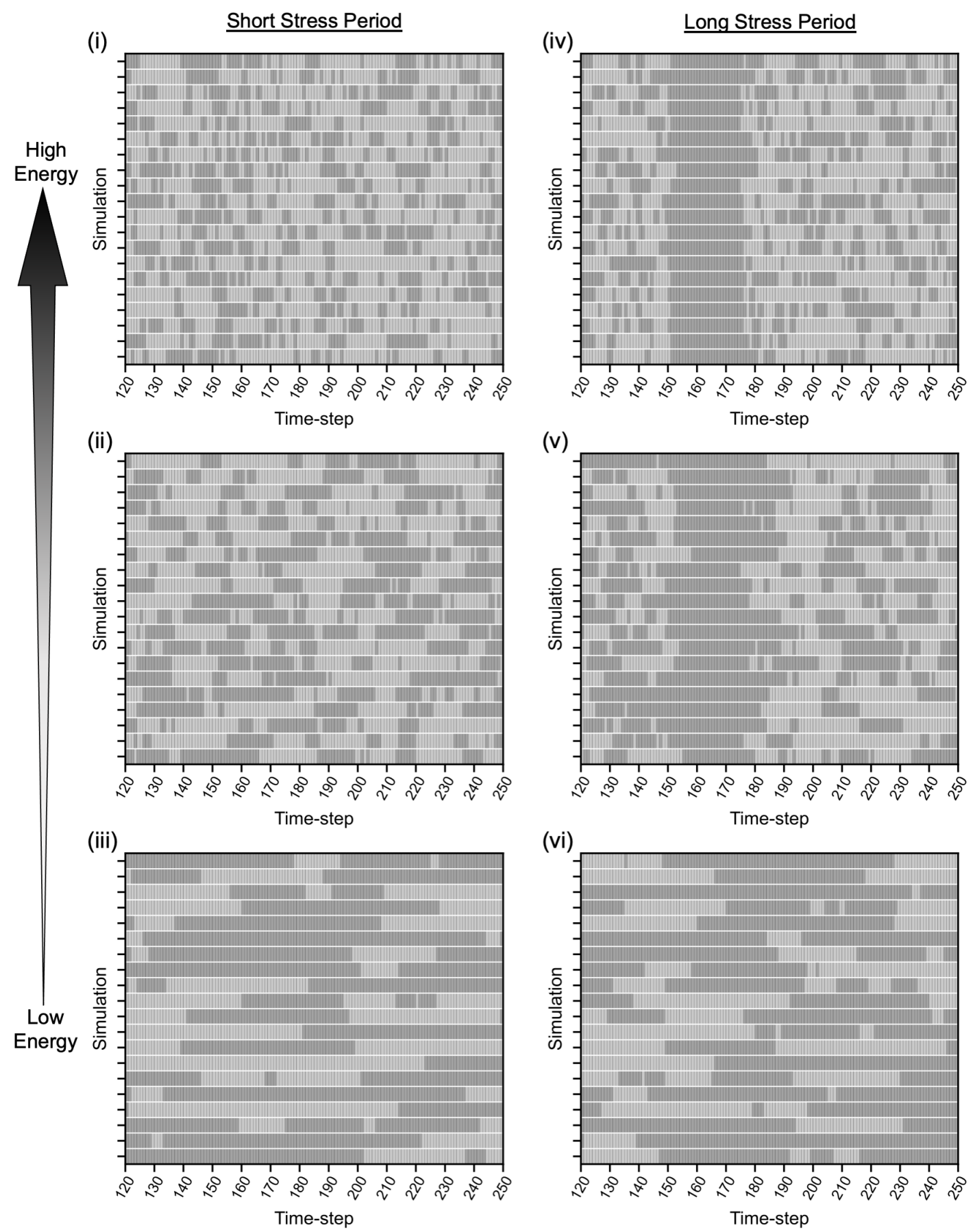

(v)
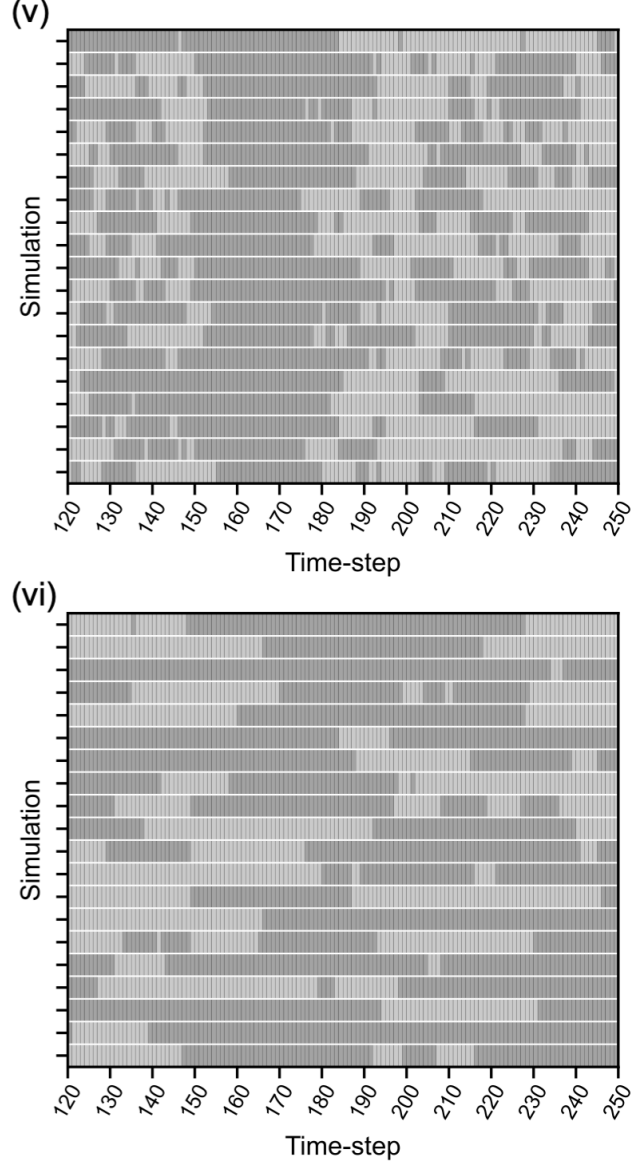

Figure 3: Simulations predict $E$. coli expresses acrAB in heterogeneous pulses in the absence of stress. Qualitative plots displaying acrAB "on"/"off" (dark/light grey) dynamics for 20 randomly selected individual E. coli simulations, when exposed to a (i)-(iii) short or (iv)-(vi) long stressor period. Panels display acrAB dynamics between timesteps $t=120-250$ at low, intermediate, and high energy availability. The short stressor period is a single timestep pulse activated at $t=150$ and the long stressor period is activated at $t=150$ until $t=174$. 
matrices describing the dynamics of the E. coli regulatory network in the presence and absence of a stressor, visualised through heatmap images (Fig. 4; see Fig. 1(A) for illustration of the process to yield these outputs).

In the absence of stress, all transitions are accessible (Fig. 4(i)). When a stress is applied, the accessible transitions for each network state are significantly restricted (compare Fig. 4 (i) to (iv)). The presence of a stressor removes the negative regulation within E. coli's regulatory architecture (Fig. 1(B)), and consequently the ability to actively switch components "off". The stressed network contains a steady-state attractor - a single state that when reached stays constant - corresponding to all components of the network being "on". Under stress-free conditions no such single steady-state attractor exists (Fig. 4 (i)). The presence of this attractor implies that an $E$. coli cell under stress shifts its regulatory poise to allow the expression of each GRN component, allowing a response to the stressor and a faster dynamic response when the stress is removed.

\subsection{Efflux network behaviour is modulated by available cellular energy}

We next sought to understand how energy availability changes network behaviour in E. coli cells, in an environment with and without a stressor. We first explored how the amount of energy available to a cell affects the accessible network transitions. Both with and without stress, the accessible transitions for each network state are the same across all energy levels, but the probabilities of these transitions vary dramatically with energy (Fig. 4). At lower energies, it is more likely that the system remains in the same state from one timestep to the next; increasing energy distributes the probability of each accessible transition more evenly amongst the total accessible transitions.

When a stressor is present, accessible transitions are restricted to subsets of the stress-free case at each energy level (Fig. 4 (i)-(iii) versus (iv)-(vi)). As energy increases, the likelihood of transitioning between supported network behaviours expands, transitioning more directly toward the steady-state attractor, '1111'. Therefore, increasing energy availability supports more rapid shifting of behaviour in the $E$. coli efflux network (Fig. 4), particularly towards a high efflux state.

Next, we asked whether the equilibrium mean expression of efflux pump genes changed at different energy levels, both in the presence and absence of stress. Perhaps counterintuitively, we found that increasing energy availability decreased the mean expression equilibrium of acr $A B$ in simulated stress-free conditions (Fig. 2). In the presence of a stressor, the population response is more rapid and of higher magnitude as energy availability increases (Fig. 2). Simulated single-cell dynamics predict cell-to-cell variability in the time taken to transition to an active efflux expression state during stress exposure in each energy state (Fig. 3). The delayed response is more pronounced as energy decreases (Fig. 3 (iv) versus (vi)). Following either stress period, the network returns to the pre-stress equilibrium more rapidly at higher energy levels (Fig. 2).

\subsection{Energy levels influence heterogeneity in individual expression level dynamics}

To investigate how energy level influences the detailed dynamics of single-cell pulses of efflux pump genes $a c r A B$, we calculated the mean pulse length in acrAB expression at different energy levels (Fig. 5). Intuitively, from time-scaling effects, mean pulse length increased as energy decreased (Fig. 5 (i)), agreeing with qualitative observations (Fig. 3 (i)-(iii)). The introduction of a short stress results in only a very small transient increase in mean pulse length, more pronounced at higher energy (Fig. 5). Pulse lengths during the long stress period are more dependent on energy level, with pulse statistics in high-energy cells changing more dramatically than in low-energy cells.

We next addressed our central hypothesis, whether energy availability could be a cause of the observed cell-to-cell variability in efflux pump expression. To explore this, we calculated the dynamics of the expression level coefficient of variation (ELCV) for E. coli acrAB at each energy level. As seen previously, substantial cell-to-cell variability exists in the system in the absence of a stressor, reflecting asynchrony and intrinsic noise in the system (Fig. 2). This variability is higher for low-energy cells (ELCV 1.34 at low 


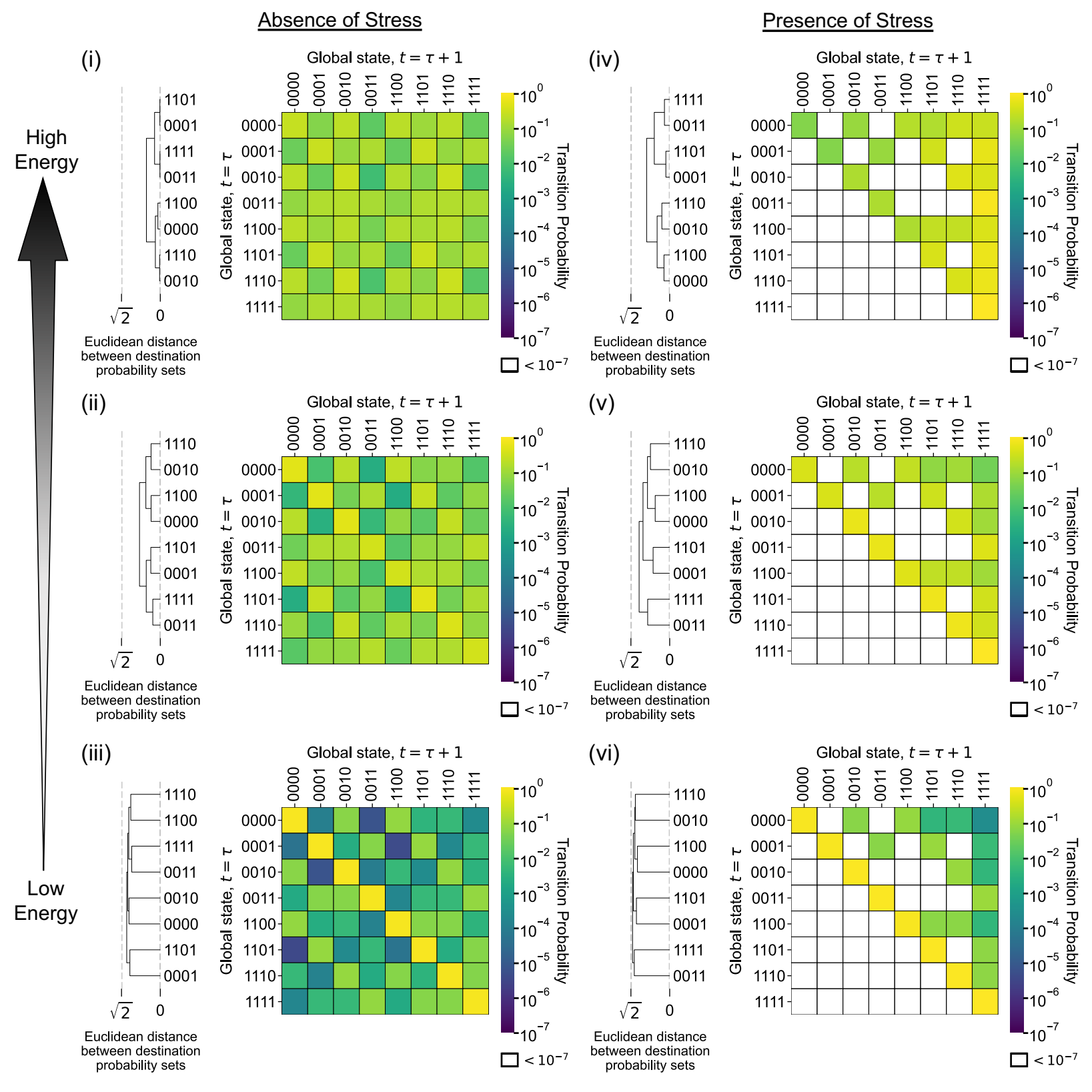

Figure 4: E. coli transition dynamics depend on cellular energy and the presence of stress. Heatmap plots showing E. coli's transition matrix. Each row contains the transition probability distribution from each of possible global states (rows) to each subsequent state (columns), in the absence (i)-(iii) and presence (iv)-(vi) of a stressor, with increasing energy availability; here, due to the coupling of marR and marA (as they form the marRA operon), we do not artificially implement unattainable network states, for example, '1011'. Each matrix element is coloured to indicate the probability $p$ (using a logarithmic scale) that global state $\mathbf{A}$ transitions to global state $\mathbf{B}$ from timestep $t=\tau$ to $t=\tau+1$. Hierarchical clustering of global states is calculated using the destination possibilities $(t=\tau+1)$ for each $t=\tau$ global state, with clustering employing a Euclidean distance metric to determine the distances between each element. Global state binary vectors are displayed in the order [marR, marA, acrR, acrAB]. 
(i)

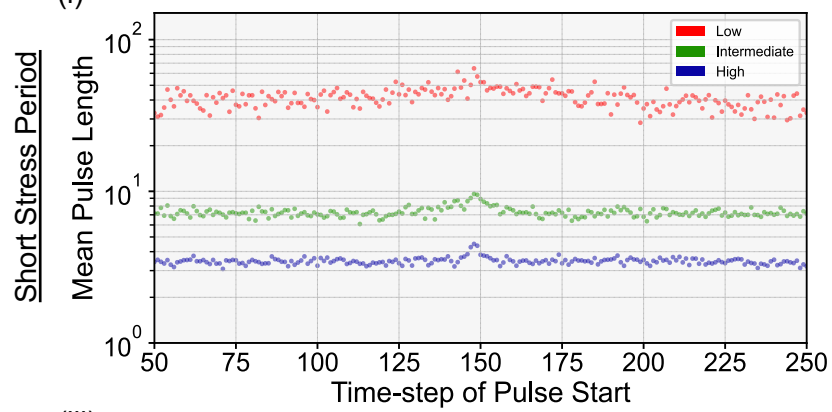

(iii)

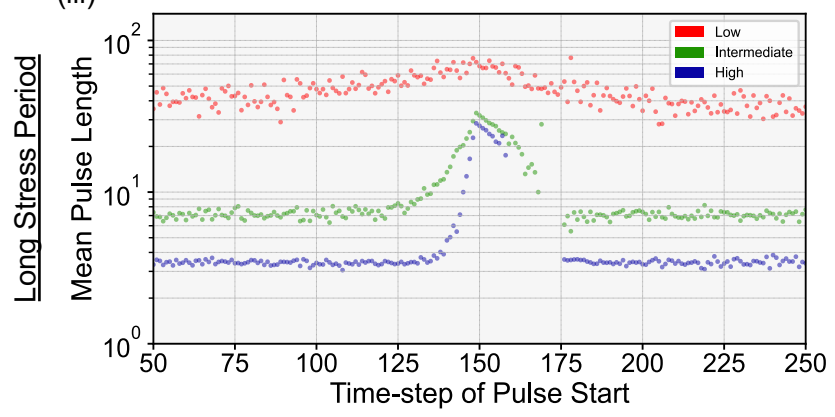

(ii)

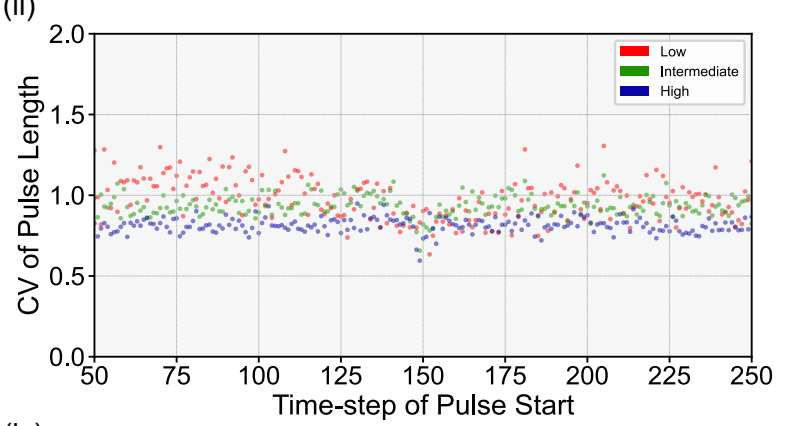

(iv)

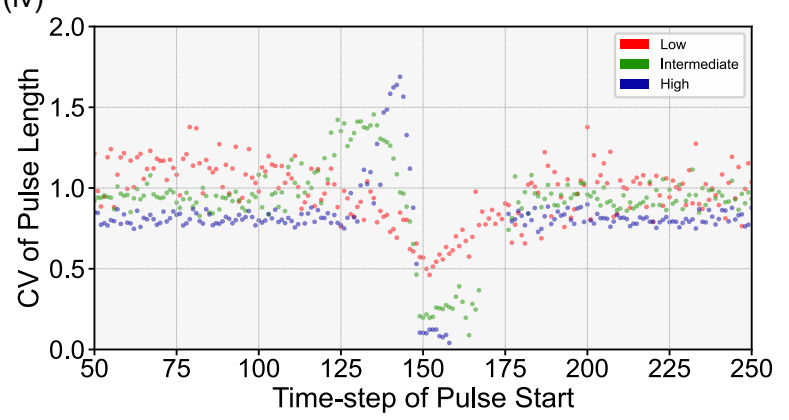

Figure 5: Energy availability and stress modulate $E$. coli acrAB pulse variability. Scatter plots displaying the (logarithmic) mean and coefficient of variation of acrAB pulse lengths (coloured dots for low (red), intermediate (green) and high (blue) energy levels) starting at each timestep in E. coli. Statistics are displayed between timesteps $t=50-250$ to observe pulse behaviour before, during and after a (i)-(ii) short or (iii)-(iv) long stress period. The short stressor period is a single timestep pulse activated at $t=150$ and the long stressor period is activated at $t=150$ until $t=174$.

energy, 1.01 at high energy). Exposure to stress reduces this variability, as the population synchronises and responds in a similar way to the imposed stressor. Exposure to a longer stress period imposed a more substantial decrease in efflux variability than a shorter stress at all energy levels (Fig. 2 (i) and (iii)) and, as observed for the maximal energy case (Section 2.4), the ELCV converged to zero at intermediate to high energy (Fig. 2(iii)). After the stress exposure, the original level of variability is recovered, allowing the same potential 'hedging' against further stress in the future (Fig. 22(i)) [55-58].

Next, we quantitatively investigated how energy level influences the variability of efflux pump gene expression pulses, by calculating the coefficient of variation of pulse lengths (PLCV) starting from each timestep in $\operatorname{acr} A B$ (Fig. 5). In the absence of stress, heterogeneity in pulse length is higher at lower energy (Fig. 5 (ii) and (iv)), whilst high energy levels display relatively low PLCV $<1$, corresponding to lower variability when dynamics are faster and individual events less rare. As with expression level dynamics, when stimulated by a short stress period, PLCV decreases, more dramatically at lower energy levels (Fig. 5 (ii)), as the population becomes more synchronised by the imposition of an external stress. For longer stress periods (Fig. 5 (iv)), differences between different energy levels are more dramatic. Pulse lengths remain highly variable for the low energy population, but PLCV decreases to zero at higher energy. Thus, higher energy availability allows more concerted and synchronous population-level behaviour in dealing with the stressor. Following stress exposure, PLCV behaviour returns to its pre-stress level for each energy level.

\subsection{E. coli and Salmonella efflux networks share functional similarity}

We next compared the behaviour of the Salmonella network to identify similarities and differences between bacterial species. We first explored the capacity of the Salmonella network to transition between expres- 
sion states. As with E. coli, the Salmonella network architecture supports the same accessible transitions (Supplementary Fig. S5). Clustering by the set of accessible transitions for each global state at a given energy level shows analogous features in both bacterial species, with transitions between states limited by energy (Supplementary Fig. S5).

Next, we asked how Salmonella's acrAB efflux pump genes respond to a stressor, compared to the homologous efflux pump in E. coli, by analysing the population-level activity of acrAB in both species in response to a short and long stress period (Fig. 22 and Supplementary Fig. S3 for E. coli and Salmonella respectively). Simulation results predict both species respond more dynamically to stress at higher energy, regardless of stress duration and, at each energy level, rapidly return to baseline conditions after the stress period (compare Fig. 2 and Supplementary Fig. S3).

Salmonella displays several other analogous behaviours compared to E. coli. At maximal energy availability, the number of timesteps for Salmonella to return to the mean expression baseline after a short and long stress period is similar (Supplementary Fig. S3), as observed for E. coli (see Section 2.3). Other analogous behaviours include a zero acrAB ELCV at the maximum stress response to the long stress period (Supplementary Fig. S3 (iii)), and the observation of heterogeneous pulses in network components (Supplementary Figs. S7 S8).

Simulations predict the stress-free acrAB ELCV is lower in Salmonella at low and intermediate energy, but greater at high energy, compared to E. coli (compare Fig. 2 (i) and Supplementary Fig. S3 (i)). This behaviour is a downstream consequence of the mean and variability in global activator expression in each species, which is modulated by the energy level, and the decoupled expression of Salmonella's activator $\operatorname{ram} A$ and repressor ramR, compared with E. coli's coupled marRA operon (Fig. 1(B)-(C)).

Together, this suggests the gene regulatory networks for both bacterial species in this study display behavioural similarities in the presence and absence of a stressor, but despite structural similarities in their architectures (Fig. 1 there are differences in the dynamics of efflux pump expression (Fig. 2 and Supplementary Fig. S3.

Table 1: Summary of energy availability effects on regulatory network behaviour. The table displays a summary of our main results from applying the modified Boolean network framework to $E$. coli and Salmonella acrAB GRNs. The effect of low, intermediate, and high energy availability on each key result is presented through a discretised scale: low $(\bullet)$, medium $(\bullet \bullet)$ and high $(\bullet \bullet \bullet)$.

\begin{tabular}{|l|c|c|c|}
\hline & $\begin{array}{c}\text { Low } \\
\text { Energy }\end{array}$ & $\begin{array}{c}\text { Intermediate } \\
\text { Energy }\end{array}$ & $\begin{array}{c}\text { High } \\
\text { Energy }\end{array}$ \\
\hline Speed of network dynamics. & $\bullet$ & $\bullet$ & $\bullet \bullet$ \\
\hline $\begin{array}{l}\text { Diversity of supported network behaviour in the ab- } \\
\text { sence of stress }\end{array}$ & $\bullet \bullet$ & $\bullet \bullet$ & $\bullet \bullet$ \\
\hline $\begin{array}{l}\text { Diversity of supported network behaviour in the } \\
\text { presence of stress }\end{array}$ & $\bullet$ & $\bullet$ & $\bullet$ \\
\hline $\begin{array}{l}\text { Cell-to-cell variability in population-level acrAB ex- } \\
\text { pression in the absence of stress. }\end{array}$ & $\bullet \bullet$ & $\bullet \bullet \bullet$ & $\bullet \bullet$ \\
\hline $\begin{array}{l}\text { Cell-to-cell variability in population-level acrAB ex- } \\
\text { pression in the presence of stress. }\end{array}$ & $\bullet$ & $\bullet$ & $\bullet$ \\
\hline $\begin{array}{l}\text { Cell-to-cell variability in pulses of acrAB expression } \\
\text { in the absence of stress. }\end{array}$ & $\bullet \bullet$ & $\bullet$ & $\bullet$ \\
\hline $\begin{array}{l}\text { Cell-to-cell variability in pulses of } a c r A B \text { expression } \\
\text { in the presence of stress. }\end{array}$ & $\bullet \bullet \bullet$ & $\bullet$ & $\bullet$ \\
\hline
\end{tabular}




\section{Discussion}

We have applied a Boolean modelling framework to coarse-grained E. coli and Salmonella efflux pump gene regulatory networks, to explore how efflux pump activity depends on the energy available to fuel the expression of efflux proteins and their regulators. We find quantitative support for our hypothesis that differences in available energy can contribute to cell heterogeneity in efflux pump expression, and dissect this heterogeneity in different mechanisms (see Table 1 for a summary).

In the absence of cell-to-cell energy variability, our model suggests (supported by experiments) that substantial intrinsic variability exists in efflux gene expression. The imposition of a population-wide stressor reduces this intrinsic variability by partially synchronising cell behaviour; stochastic effects after stress exposure recover the original variability. This intrinsic noise can potentially be acting to hedge the population against future stress, while maintaining cells that invest less resource in defence mechanisms and/or protect the lineage from diverse environmental conditions. When extrinsic differences in ATP availability between cells are additionally imposed, the population variability in efflux response will be amplified. Cells with lower energy levels will experience more variable expression of efflux components (amplifying intrinsic variability), slower responses to stressors, and hence slower removal of stressors from the environment. In future, experimental tests of this hypothesis could involve, for example, jointly tracking bacterial ATP levels and efflux gene expression in a culture prior to, during and post antibiotic stress using fluorescent reporters $[11,12,16,59]$.

In our model, stress-free bacteria naturally express heterogeneous pulses of efflux genes (Fig. 3 and Supplementary Fig. S7). This behaviour may be biologically valuable for several reasons. The products of $a c r A B$ have an extremely long half-life in $E$. coli [60], suggesting that the genes may only need to be transcribed in short pulses (which is predicted to be a less energetically demanding process compared to translation [61]) for the protein to be in an abundant supply. Dealing with external stressors is one role of the AcrAB-TolC efflux pump, but it also, for example, removes endogenous waste products [62], suggesting the pulse behaviour could aid physiological functions by expressing acrAB mRNA to be used in these processes. The pulse behaviour may also confer a natural resistance phenotype to a fraction of cells in stress-free environmental conditions at all times, as reported experimentally in Salmonella enterica [13]. Therefore, the predicted pulsing behaviour may contribute to several essential intracellular functions.

Recent mathematical models have studied the mar network at the protein level, suggesting the motif is monostable, and supports a single deterministic steady-state in the absence of stress [63,64]. A theoretical study has also shown, at the protein level, the development of stochastic pulses in marRA products [64]. The cell-to-cell heterogeneity in mar expression that we predict agrees with ODE models of the mar operon - which account for the inherent stochasticity of biological systems - and experimental demonstrations of heterogeneous cell-to-cell steady-state mar expression within a population, in the presence and absence of stress [64-66] (Fig. 2 and Supplementary Fig. S6). With the development of advanced experimental assays, future studies may resolve the behaviour at the transcript level to test our theoretical predictions.

Even for these relatively simple networks, our Boolean modelling approach has demonstrated general principles of energy influence without requiring characterisation of a large parameter space. Developed with a view to scalability, this approach can be applied to many naturally-occurring decision-making phenomena. Our previous work [14] has demonstrated the profound influence that energy variability can have on simple decision-making circuits; this GRN approach allows the expansion of this philosophy to a much wider range of architectures, including the broader regulatory context of these core networks (see Supplementary Information sections 5.1 5.2). As cell-to-cell variability in ATP is increasingly elucidated [18-20] we believe that our approach will help reveal the theoretical underpinnings of this important influence on regulatory dynamics.

Our hypothesised relationship between energy availability and efflux-pump activity, variability, and capacity to respond to antimicrobials has, as far as we are aware, not been previously considered. However, our hypothesis is not restricted to this AMR mechanism or motif. A connected phenomenon that may be mechanistically related is the formation of bacterial persisters in the presence of antibiotics $[55,67,68]$. Here, phenotypic variants display a transient tolerance to external stress $[69,70]$, causing a leading factor in 
the recalcitrance of chronic infections [71-74]. An approach grounded in optimal control theory has found that the persister strategy is dependent upon stochastic fluctuations in both the proportion of persisters in the colony and the environment [75]. The results also predict that producing persisters in the absence of environmental volatility leads to a lower per-capita growth rate, giving the population a possible evolutionary disadvantage. Increasing evidence has reported a mechanism linking persister cells and intracellular energy, or more specifically ATP [17,76-79]. One study has gone further, suggesting there may be a general low energy mechanism of persister formation in bacteria [80]. This relationship bears substantial similarities to our model where we predict high variability and noise in gene expression pulses in low energy cells, compared to intermediate and high energy cells (Fig. 22and Fig. 5). High expression variability contributes to cell-to-cell variation within a clonal population, implying a different set of phenotypes may be expressed in the low energy sub-population. As energy, or more precisely ATP, increases, cell-to-cell variability decreases, implying a uniform phenotype displayed across the population. We would predict, in agreement with growing evidence, intracellular ATP is a factor in the mechanisms of persister generation, and future work will explore this using our approach when the governing GRNs can be identified.

\section{Methods}

\subsection{Wiring Diagrams}

In the supplement (Supplementary Fig. S1 we present the wiring diagram form of each gene regulatory network (Fig. 1(B)-(C)). The wiring diagrams contain the regulatory interactions in each GRN (activatory or inhibitory) and edge weights, outputting a directed graph. A stress, such as antimicrobial exposure, is included through the 'stress node', $S$, within the wiring diagram. A stress input is modelled as repressing gene-gene regulatory edges, representing a 'repressive' modulation of transcription factors (MarR/RamR and AcrR). The capacity to expel substances by the efflux pump is integrated through a repressive regulation from acrAB to $S$.

Estimates of edge weights were gleaned from the relevant literature, integrating the natural behaviour of each network, and giving each regulatory actor the ability to operate. In $E$. coli, the marRA operon is separated into its individual components to allow each regulation of marRA to be captured, but are coupled in all iterative updates; the inability to model a node that both positively and negatively regulates itself is a limitation of the Boolean modelling framework. Experimental data has shown transcription of marA and acrA increase in marR mutant cells [81], and MICs of various antibiotics increase in an acrR mutant strain [44] (consistent with the limited repressor function of AcrR [41]). Together, these suggest, under under normal conditions, edge weights of interactions from marR to marRA and acrR to acrAB should be dominant. Inactivation of the ramR gene upstream of $\operatorname{ram} A$ resulted in increased expression of $\operatorname{ram} A$ and the AcrAB efflux pump in Salmonella [82]. This requires the edge weight from $\operatorname{ram} R$ to $\operatorname{ram} A$ to outweigh $\operatorname{ram} A$ basal expression under normal conditions. Constitutive gene expression is captured using 'ghost nodes'. These nodes are permanently "on" in the network, supplying a constant positive influx to a node.

Interaction matrix $\mathrm{J}$ is constructed from the data contained within a wiring diagram. A weighted edge from node $j$ to node $i$, is represented by element $\mathrm{J}_{i, j}$ within $\mathrm{J}$. Each matrix element can take a positive, negative or zero value; edge weight sign corresponds to a negative, positive or absence of regulation respectively. Since edges are directed, $\mathrm{J}_{i, j} \neq \mathrm{J}_{j, i}$, except for $i=j$ or $\mathrm{J}_{i, j}=\mathrm{J}_{j, i}=0$. Matrix $\mathrm{J}$ is implemented in numerical simulations of the Boolean model and has dimension $N$-by- $N$ ( $N$ is the total number of nodes in a wiring diagram, including ghost and stress nodes).

\subsection{Initial conditions}

Two configurations of initial node states were considered in simulations of the E. coli and Salmonella wiring diagrams. These were named the "unstressed" and "stressed" initial condition, with the initial node states set as follows: 
Table 2: $\boldsymbol{E}$. coli node initial values. Table defines the stressed and unstressed initial conditions for $E$. coli gene regulatory network components (Fig. $1(\mathrm{~B})$ ).

\begin{tabular}{|l|c|c|c|c|}
\hline & marR & marA & acrR & acrAB \\
\hline Unstressed & 0 & 0 & 1 & 0 \\
\hline Stressed & 1 & 1 & 0 & 1 \\
\hline
\end{tabular}

Table 3: Salmonella node initial values. Table defines the stressed and unstressed initial conditions for Salmonella gene regulatory network components (Fig. $1(\mathrm{C})$ ).

\begin{tabular}{|l|c|c|c|c|}
\hline & $\operatorname{ramR}$ & $\operatorname{ram} A$ & $\operatorname{acr} R$ & $\operatorname{acr} A B$ \\
\hline Unstressed & 1 & 0 & 1 & 0 \\
\hline Stressed & 0 & 1 & 0 & 1 \\
\hline
\end{tabular}

\subsection{Modulation of transition rates allows energy variability to be captured in GRN models}

Wiring diagrams, consisting of nodes and edges, schematically represent the interactions between elements within regulatory networks (as shown in Supplementary Fig. S1). The edges often illustrate combined processes such as transcription, translation and phosphorylation. However, the information contained within edges in regulatory networks is coarse-grained, omitting a substantial amount of important biological detail. Several of the processes represented by these edges require ATP as an energy source [29, 53], so there exists a core energy dependence in the dynamics modelled within each wiring diagram.

To address this and capture the fact that all the processes involved in gene expression depend on ATP, we constructed a modelling approach modulating the strength of regulation from node $X$ to node $Y$ according to cellular energy levels. This reflects the fact that, for example, cells with low ATP will have a lower rate of transcription elongation per timestep [53]. We interpret this modulation as influencing the rate with which regulatory interactions can be applied from node $X$ to node $Y$. The rationale for this picture is that if the expression of regulatory components is slowed by reduced energy levels, the corresponding biochemical interactions will occur at a lower rate.

In the absence of energy variability, our asynchronous Boolean modelling framework interprets regulatory interactions as stochastic events occurring with a characteristic mean rate of one per timestep per interaction. To include the influence of energy availability on the system, we allow this rate to be a function of energy level. To capture an order-of-magnitude range across cells, we use rates for simulation timestep of 1 for 'high' energy (hence, all interactions are always realised), 0.5 for 'intermediate' energy, and 0.1 for 'low' energy. We thus modulate the probability of any given interaction being realised in a given simulation timestep by the level of available cellular energy.

\subsection{Node update function}

Nodes within the Boolean network are updated iteratively, using an update function. An update function defines the value of each node according to its regulatory nodes and remains fixed for all timesteps [83]. We consider a threshold update function to update the state of node $i$ at timestep $t=\tau+1$, denoted $\sigma_{i}(\tau+1)$. The argument of the update function consists of the regulatory nodes of node $i$, the states of these nodes at $t=\tau$ and the $N$-by- $N$ interaction matrix J, where $N$ is the total number of nodes in the wiring diagram. The summation value determines the state of node $i$ at $t=\tau+1$.

During an active stress time frame, the state of stress node $S$ (Supplementary Fig. $S 1$ ) is imposed to be "on" at the start of each timestep, regardless of the behaviour from the previous update. Once the final timestep within the stress period is reached, $S$ is allowed to be switched "off" from regulation within the network. 
We consider the update function displayed in Equation (4.1). Here, a node requires a non-zero sum of its inputs to change state from $t=\tau$ to $t=\tau+1$. In the case where the sum is zero, the node state remains unchanged.

$$
\sigma_{i}(\tau+1)= \begin{cases}1 & \text { if } \sum_{j=1}^{N}\left(\mathrm{~J}_{i, j} \cdot \sigma_{j}(\tau)\right)>0, \\ \sigma_{i}(\tau) & \text { if } \sum_{j=1}^{N}\left(\mathrm{~J}_{i, j} \cdot \sigma_{j}(\tau)\right)=0, \\ 0 & \text { if } \sum_{j=1}^{N}\left(\mathrm{~J}_{i, j} \cdot \sigma_{j}(\tau)\right)<0 .\end{cases}
$$

Alternate threshold update functions are possible. We could, for example, consider the conditions that set a node as "on" if there is an overall positive input, and "off" otherwise. However, this would automatically remove a stress in the system by setting the stress node state to "off" once reaching the end-point of the stress time frame. This removes the capability of the efflux node to 'expel' the stress through feedback to the stress node $S$ (Supplementary Fig. S1), behaviour which is not the focus of this study. By choosing update rule 4.1, it allows the efflux pump node to have the capacity to remove stress in the network, through regulatory interactions, depicting the behaviour observed in bacteria.

At each timestep, the binary vector containing all node states is the global, or network, state [84]. The set of all possible global states forms the state space of a network [83]. The state space for each network in this study is of size $2^{M}$ for Salmonella, as we are interested in the dynamics of the $M$-component GRN network being modelled, and $2^{M-1}$ for $E$. coli, due to the coupling of marR and marA.

\subsection{Node update methods}

There are two methods for updating global states, synchronous and asynchronous. In the synchronous mode, all the nodes (including the stress node) are updated at every timestep, with the state of each node at $t=\tau+1$ depending on the states of its inputs at $t=\tau$. The deterministic nature of synchronous Boolean models, combined with each model containing a finite state space $\left(2^{M}\right.$ or $\left.2^{M-1}\right)$, where $M$ is the number of elements in the original GRN, guarantees that a synchronously updated time series simulation will eventually end at a steady-state attractor, or a cycle of repeating global states [83]. It also means the network will always reach the same state for a given initial condition and number of timesteps. We do not consider synchronous updating in this study due to the artefacts that can arise from its imposition, and its inability to model stochastic processes. A more complex, but biologically realistic, protocol is the asynchronous mode, which gives a reasonable overview of the random dynamics in the cell and better captures transient behaviour. This method updates nodes of the system according to the last update of their regulator nodes, either from the previous or current iteration [85]. Previous studies have employed numerous different versions of asynchronous updating [27,86-88], including methods where all nodes are updated according to a random sequence, or one randomly selected node is updated at a time step [89]. In this study the asynchronous protocol updates $\eta$ nodes per timestep, with $\eta$ drawn from a Poisson distribution with a mean equal to the number of original elements in the regulatory network $(M)$, plus the stress node. Additionally, each node can be updated more than once per timestep and the state of each node at $t=\tau+1$ depends on the states of its inputs at the most recent update. We used an asynchronous protocol because we believe it better captures the stochasticity in the cell.

\subsection{Boolean Analysis}

Taken together, the wiring diagram, initial conditions, update functions and update method form the Boolean network model. Time-dependent numerical solutions of the presented Boolean model were generated in Python 3.8.2 using modules numpy, pandas and random. Numerical solutions were calculated from prescribed initial conditions covering the stressed and unstressed state of each network. Time-series figures, displaying the expression profiles of each node, were constructed from the mean of node expression and standard deviation per timestep, from $10^{4}$ simulations. Visualisations of 'single-cell' expression 
profiles, and pulse statistics, were constructed from 20 and 5000 randomly selected simulations respectively. Transition matrices were constructed from the simulation data and visualised through a heatmap structure, from $10^{7}$ simulations. Each entry of the transition matrix is a non-negative real number representing the probability of transitioning between two network states from timestep $t=\tau$ to $t=\tau+1$. Clustering of global states was performed using the same data, with a Euclidean distance matrix from modules scipy.cluster and scipy.cluster.hierarchy. All scripts used in this study are openly accessible through https://github.com/StochasticBiology/boolean-efflux.git.

\section{Acknowledgements}

RK thanks the Wellcome Trust for funding (grant reference 108876/Z/15/Z).

\section{References}

[1] Review on Antimicrobial Resistance. Tackling drug-resistant infections globally: final report and recommendations. Review on antimicrobial resistance, 2016.

[2] JIM O'neill. Antimicrobial resistance: tackling a crisis for the health and wealth of nations. Rev. Antimicrob. Resist, 20:1-16, 2014.

[3] Hiroshi Nikaido. Multidrug efflux pumps of gram-negative bacteria. J Bacteriol, 178(20):5853, 1996.

[4] Hiroshi Nikaido and Jean-Marie Pagès. Broad-specificity efflux pumps and their role in multidrug resistance of gram-negative bacteria. FEMS microbiology reviews, 36(2):340-363, 2012.

[5] K Poole. Efflux-mediated multiresistance in gram-negative bacteria. Clinical Microbiology and infection, 10(1):12-26, 2004.

[6] Olga Lomovskaya et al. Identification and characterization of inhibitors of multidrug resistance efflux pumps in pseudomonas aeruginosa: novel agents for combination therapy. Antimicrobial agents and chemotherapy, 45(1):105-116, 2001.

[7] Penelope N Markham et al. Multiple novel inhibitors of the nora multidrug transporter of staphylococcus aureus. Antimicrobial agents and chemotherapy, 43(10):2404-2408, 1999.

[8] Mark L Nelson and Stuart B Levy. Reversal of tetracycline resistance mediated by different bacterial tetracycline resistance determinants by an inhibitor of the tet (b) antiport protein. Antimicrobial agents and chemotherapy, 43(7):1719-1724, 1999.

[9] Rafael Pereira da Cruz et al. Effect of $\alpha$-bisabolol and its $\beta$-cyclodextrin complex as tetk and nora efflux pump inhibitors in staphylococcus aureus strains. Antibiotics, 9(1):28, 2020.

[10] Niranjana Sri Sundaramoorthy et al. Restoring colistin sensitivity in colistin-resistant e. coli: combinatorial use of marr inhibitor with efflux pump inhibitor. Scientific Reports, 9(1):1-13, 2019.

[11] Imane El Meouche and Mary J Dunlop. Heterogeneity in efflux pump expression predisposes antibioticresistant cells to mutation. Science, 362(6415):686-690, 2018.

[12] Laura Paixão et al. Fluorometric determination of ethidium bromide efflux kinetics in escherichia coli. Journal of biological engineering, 3(1):18, 2009.

[13] María Antonia Sánchez-Romero and Josep Casadesús. Contribution of phenotypic heterogeneity to adaptive antibiotic resistance. Proceedings of the National Academy of Sciences, 111(1):355-360, 2014. 
[14] Ryan Kerr, Sara Jabbari, and lain G Johnston. Intracellular energy variability modulates cellular decision-making capacity. Scientific reports, 9(1):1-12, 2019.

[15] lain G Johnston et al. Mitochondrial variability as a source of extrinsic cellular noise. PLoS computational biology, 8(3):e1002416, 2012.

[16] Hideyuki Yaginuma et al. Diversity in atp concentrations in a single bacterial cell population revealed by quantitative single-cell imaging. Scientific reports, 4:6522, 2014.

[17] Eliza A Zalis et al. Stochastic variation in expression of the tricarboxylic acid cycle produces persister cells. mBio, 10(5):e01930-19, 2019.

[18] Masak Takaine et al. Reliable imaging of atp in living budding and fission yeast. J Cell Sci, pages jcs-230649, 2019.

[19] Tomoki Yoshida, Akira Kakizuka, and Hiromi Imamura. Bteam, a novel bret-based biosensor for the accurate quantification of atp concentration within living cells. Scientific reports, 6:39618, 2016.

[20] Valentina De Col et al. Atp sensing in living plant cells reveals tissue gradients and stress dynamics of energy physiology. Elife, 6:e26770, 2017.

[21] Stuart A Kauffman. Metabolic stability and epigenesis in randomly constructed genetic nets. Journal of theoretical biology, 22(3):437-467, 1969.

[22] René Thomas. Boolean formalization of genetic control circuits. Journal of theoretical biology, 42(3):563-585, 1973.

[23] Fangting $\mathrm{Li}$ et al. The yeast cell-cycle network is robustly designed. Proceedings of the National Academy of Sciences, 101(14):4781-4786, 2004.

[24] Aurélien Naldi, Jorge Carneiro, Claudine Chaouiya, and Denis Thieffry. Diversity and plasticity of th cell types predicted from regulatory network modelling. PLoS computational biology, 6(9), 2010.

[25] Réka Albert and Hans G Othmer. The topology of the regulatory interactions predicts the expression pattern of the segment polarity genes in drosophila melanogaster. Journal of theoretical biology, 223(1):1-18, 2003.

[26] Manuel Banzhaf, Osbaldo Resendis-Antonio, and M Lisandra Zepeda-Mendoza. Uncovering the dynamic mechanisms of the pseudomonas aeruginosa quorum sensing and virulence networks using boolean modelling. IEEE Transactions on NanoBioscience, 2020.

[27] Stylianos E Dallidis and loannis G Karafyllidis. Boolean network model of the pseudomonas aeruginosa quorum sensing circuits. IEEE transactions on nanobioscience, 13(3):343-349, 2014.

[28] Violeta Balbas-Martinez et al. A systems pharmacology model for inflammatory bowel disease. PloS one, 13(3), 2018.

[29] Moshe Kafri, Eyal Metzl-Raz, Ghil Jona, and Naama Barkai. The cost of protein production. Cell reports, 14(1):22-31, 2016.

[30] Martin J Everett, Yu Fang Jin, Vito Ricci, and LJ Piddock. Contributions of individual mechanisms to fluoroquinolone resistance in 36 escherichia coli strains isolated from humans and animals. Antimicrobial agents and chemotherapy, 40(10):2380-2386, 1996.

[31] Annarita Mazzariol et al. High-level fluoroquinolone-resistant clinical isolates of escherichia coli overproduce multidrug efflux protein acra. Antimicrobial agents and chemotherapy, 44(12):3441-3443, 2000. 
[32] Mark A Webber and Laura JV Piddock. Absence of mutations in marrab or soxrs in acrb-overexpressing fluoroquinolone-resistant clinical and veterinary isolates of escherichia coli. Antimicrobial agents and chemotherapy, 45(5):1550-1552, 2001.

[33] Sylvie Baucheron et al. Acrab-tolc directs efflux-mediated multidrug resistance in salmonella enterica serovar typhimurium dt104. Antimicrobial agents and chemotherapy, 48(10):3729-3735, 2004.

[34] Deborah J Eaves, Vito Ricci, and Laura JV Piddock. Expression of acrb, acrf, acrd, mara, and soxs in salmonella enterica serovar typhimurium: role in multiple antibiotic resistance. Antimicrobial agents and chemotherapy, 48(4):1145-1150, 2004.

[35] Laura JV Piddock et al. Evidence for an efflux pump mediating multiple antibiotic resistance in salmonella enterica serovar typhimurium. Antimicrobial Agents and Chemotherapy, 44(11):31183121, 2000.

[36] Vito Ricci et al. Ciprofloxacin-resistant salmonella enterica serovar typhimurium strains are difficult to select in the absence of acrb and tolc. Antimicrobial Agents and Chemotherapy, 50(1):38-42, 2006.

[37] Elena B Tikhonova and Helen I Zgurskaya. Acra, acrb, and tolc of escherichia coli form a stable intermembrane multidrug efflux complex. Journal of Biological Chemistry, 279(31):32116-32124, 2004.

[38] Helen I Zgurskaya, Ganesh Krishnamoorthy, Abigail Ntreh, and Shuo Lu. Mechanism and function of the outer membrane channel tolc in multidrug resistance and physiology of enterobacteria. Frontiers in microbiology, 2:189, 2011.

[39] Seth P Cohen, H Hächler, and SB Levy. Genetic and functional analysis of the multiple antibiotic resistance (mar) locus in escherichia coli. Journal of bacteriology, 175(5):1484-1492, 1993.

[40] Eiji Nikaido, Akihito Yamaguchi, and Kunihiko Nishino. Acrab multidrug efflux pump regulation in salmonella enterica serovar typhimurium by rama in response to environmental signals. Journal of Biological Chemistry, 283(35):24245-24253, 2008.

[41] Dzwokai Ma et al. The local repressor acrr plays a modulating role in the regulation of acrab genes of escherichia coli by global stress signals. Molecular microbiology, 19(1):101-112, 1996.

[42] Dzwokai Ma et al. Genes acra and acrb encode a stress-induced efflux system of escherichia coli. Molecular microbiology, 16(1):45-55, 1995.

[43] Joe A Fralick. Evidence that tolc is required for functioning of the mar/acrab efflux pump of escherichia coli. Journal of bacteriology, 178(19):5803-5805, 1996.

[44] Haruko Okusu, Dzwokai Ma, and Hiroshi Nikaido. Acrab efflux pump plays a major role in the antibiotic resistance phenotype of escherichia coli multiple-antibiotic-resistance (mar) mutants. Journal of bacteriology, 178(1):306-308, 1996.

[45] Xian-Zhi Li, Patrick Plésiat, and Hiroshi Nikaido. The challenge of efflux-mediated antibiotic resistance in gram-negative bacteria. Clinical microbiology reviews, 28(2):337-418, 2015.

[46] David G Thanassi, Luisa W Cheng, and Hiroshi Nikaido. Active efflux of bile salts by escherichia coli. Journal of bacteriology, 179(8):2512-2518, 1997.

[47] Michael N Alekshun and Stuart B Levy. Alteration of the repressor activity of marr, the negative regulator of the escherichia coli marrab locus, by multiple chemicals in vitro. Journal of bacteriology, 181(15):4669-4672, 1999.

[48] Sylvie Baucheron et al. Bile-mediated activation of the acrab and tolc multidrug efflux genes occurs mainly through transcriptional derepression of rama in salmonella enterica serovar typhimurium. Journal of Antimicrobial Chemotherapy, 69(9):2400-2406, 2014. 
[49] AJ Lawler, V Ricci, SJW Busby, and LJV Piddock. Genetic inactivation of acrab or inhibition of efflux induces expression of rama. Journal of Antimicrobial Chemotherapy, 68(7):1551-1557, 2013.

[50] Robert G Martin and Judah L Rosner. Binding of purified multiple antibiotic-resistance repressor protein (marr) to mar operator sequences. Proceedings of the National Academy of Sciences, 92(12):5456-5460, 1995.

[51] Chih-Chia Su, Denae J Rutherford, and W Yu Edward. Characterization of the multidrug efflux regulator acrr from escherichia coli. Biochemical and biophysical research communications, 361(1):85-90, 2007.

[52] Suguru Yamasaki et al. The crystal structure of multidrug-resistance regulator ramr with multiple drugs. Nature communications, 4(1):1-7, 2013.

[53] Ricardo Pires Das Neves, Nick S Jones, Lorena Andreu, Rajeev Gupta, Tariq Enver, and Francisco J Iborra. Connecting variability in global transcription rate to mitochondrial variability. PLoS biology, 8(12):e1000560, 2010.

[54] Mark C Sulavik, Laura F Gambino, and Paul F Miller. The marr repressor of the multiple antibiotic resistance (mar) operon in escherichia coli: prototypic member of a family of bacterial regulatory proteins involved in sensing phenolic compounds. Molecular Medicine, 1(4):436-446, 1995.

[55] Nathalie Q Balaban et al. Bacterial persistence as a phenotypic switch. Science, 305(5690):16221625, 2004.

[56] Sasha F Levy, Naomi Ziv, and Mark L Siegal. Bet hedging in yeast by heterogeneous, age-correlated expression of a stress protectant. PLoS Biol, 10(5):e1001325, 2012.

[57] lain G Johnston and George W Bassel. Identification of a bet-hedging network motif generating noise in hormone concentrations and germination propensity in arabidopsis. Journal of The Royal Society Interface, 15(141):20180042, 2018.

[58] Jan-Willem Veening, Leendert W Hamoen, and Oscar P Kuipers. Phosphatases modulate the bistable sporulation gene expression pattern in bacillus subtilis. Molecular microbiology, 56(6):1481-1494, 2005.

[59] Tobias Bergmiller et al. Biased partitioning of the multidrug efflux pump acrab-tolc underlies long-lived phenotypic heterogeneity. Science, 356(6335):311-315, 2017.

[60] Qian Chai et al. Study of the degradation of a multidrug transporter using a non-radioactive pulse chase method. Analytical and bioanalytical chemistry, 408(27):7745-7751, 2016.

[61] Michael Lynch and Georgi K Marinov. The bioenergetic costs of a gene. Proceedings of the National Academy of Sciences, 112(51):15690-15695, 2015.

[62] Paula Blanco et al. Bacterial multidrug efflux pumps: much more than antibiotic resistance determinants. Microorganisms, 4(1):14, 2016.

[63] Guillermo Rodrigo, Djordje Bajic, Ignacio Elola, and Juan F Poyatos. Antagonistic autoregulation speeds up a homogeneous response in escherichia coli. Scientific reports, 6:36196, 2016.

[64] Javier Garcia-Bernardo and Mary J Dunlop. Tunable stochastic pulsing in the escherichia coli multiple antibiotic resistance network from interlinked positive and negative feedback loops. PLoS Comput Biol, 9(9):e1003229, 2013.

[65] Guillermo Rodrigo, Djordje Bajić, Ignacio Elola, and Juan F Poyatos. Deconstructing a multiple antibiotic resistance regulation through the quantification of its input function. NPJ systems biology and applications, 3(1):1-9, 2017. 
[66] Imane El Meouche, Yik Siu, and Mary J Dunlop. Stochastic expression of a multiple antibiotic resistance activator confers transient resistance in single cells. Scientific reports, 6(1):1-9, 2016.

[67] Joseph W Bigger et al. Treatment of staphylococcal infections with penicillin by intermittent sterilisation. Lancet, pages 497-500, 1944.

[68] Samara PM Drescher et al. Salmonella enterica persister cells form unstable small colony variants after in vitro exposure to ciprofloxacin. Scientific reports, 9(1):7232, 2019.

[69] Edo Kussell, Roy Kishony, Nathalie Q Balaban, and Stanislas Leibler. Bacterial persistence: a model of survival in changing environments. Genetics, 169(4):1807-1814, 2005.

[70] Kim Lewis. Persister cells. Annual review of microbiology, 64:357-372, 2010.

[71] Maarten Fauvart, Valerie N De Groote, and Jan Michiels. Role of persister cells in chronic infections: clinical relevance and perspectives on anti-persister therapies. Journal of medical microbiology, 60(6):699-709, 2011.

[72] Michael D LaFleur, Qingguo Qi, and Kim Lewis. Patients with long-term oral carriage harbor highpersister mutants of candida albicans. Antimicrobial agents and chemotherapy, 54(1):39-44, 2010.

[73] Lawrence R Mulcahy, Jane L Burns, Stephen Lory, and Kim Lewis. Emergence of pseudomonas aeruginosa strains producing high levels of persister cells in patients with cystic fibrosis. Journal of bacteriology, 192(23):6191-6199, 2010.

[74] Ying Zhang, Wing Wai Yew, and Michael R Barer. Targeting persisters for tuberculosis control. Antimicrobial agents and chemotherapy, 56(5):2223-2230, 2012.

[75] Alexander P Browning et al. Persistence as an optimal hedging strategy. Biophysical Journal, 2020.

[76] Brian P Conlon et al. Persister formation in staphylococcus aureus is associated with atp depletion. Nature microbiology, 1(5):1-7, 2016.

[77] Xiaofan Liu et al. The potassium transporter kdpa affects persister formation by regulating atp levels in mycobacterium marinum. Emerging Microbes \& Infections, 9(1):129-139, 2020.

[78] Dan L Nabb et al. Polymicrobial interactions induce multidrug tolerance in staphylococcus aureus through energy depletion. Frontiers in Microbiology, 10:2803, 2019.

[79] Yue Shan et al. Atp-dependent persister formation in escherichia coli. MBio, 8(1):e02267-16, 2017.

[80] Sylvie Manuse et al. Bacterial persisters are a stochastically formed subpopulation of low-energy cells. PLOS Biology, 19(4):1-18, 042021.

[81] Hervé Nicoloff, Vincent Perreten, Laura M McMurry, and Stuart B Levy. Role for tandem duplication and lon protease in acrab-tolc-dependent multiple antibiotic resistance (mar) in an escherichia coli mutant without mutations in marrab or acrrab. Journal of bacteriology, 188(12):4413-4423, 2006.

[82] Yousef M Abouzeed, Sylvie Baucheron, and Axel Cloeckaert. ramr mutations involved in effluxmediated multidrug resistance in salmonella enterica serovar typhimurium. Antimicrobial agents and chemotherapy, 52(7):2428-2434, 2008.

[83] Florian Greil. Boolean networks as modeling framework. Frontiers in plant science, 3:178, 2012.

[84] Guy Karlebach and Ron Shamir. Modelling and analysis of gene regulatory networks. Nature Reviews Molecular Cell Biology, 9(10):770-780, 2008. 
[85] Itziar Irurzun-Arana, José Martín Pastor, Iñaki F Trocóniz, and José David Gómez-Mantilla. Advanced boolean modeling of biological networks applied to systems pharmacology. Bioinformatics, 33(7):1040-1048, 2017.

[86] Madalena Chaves, Eduardo D Sontag, and Réka Albert. Methods of robustness analysis for boolean models of gene control networks. IEE Proceedings-Systems Biology, 153(4):154-167, 2006.

[87] Inman Harvey and Terry Bossomaier. Time out of joint: Attractors in asynchronous. In Fourth European Conference on Artificial Life, volume 4, page 67. MIT Press, 1997.

[88] Rui-Sheng Wang, Assieh Saadatpour, and Reka Albert. Boolean modeling in systems biology: an overview of methodology and applications. Physical biology, 9(5):055001, 2012.

[89] Assieh Saadatpour and Réka Albert. Boolean modeling of biological regulatory networks: a methodology tutorial. Methods, 62(1):3-12, 2013. 


\section{Supplementary Information}

\subsection{A Bottom-up Construction of Efflux Pump Gene Regulatory Networks}

A key regulator of efflux-related drug resistance in $E$. coli is the multiple antibiotic resistance (mar) operon $[1,2]$. This genomic region contains the marRAB operon, encoding three proteins named MarR, MarA and MarB [1]. MarR has a repressive function, acting on the basal expression of the mar operon in stressfree conditions $[1,3,4]$. MarA, the marA gene product, is a member of the AraC/XylS family of proteins which induces acrAB expression and undergoes positive feedback through auto-activation of the marRAB operon [5, 6]; in addition to $\operatorname{acr} A B$ and marRAB, MarA regulates the expression of numerous other genes, either directly or indirectly, in its regulon [7]. The final product from the operon, MarB, is a small periplasmic protein that represses marRAB but does so through an unknown mechanism and is therefore omitted from our regulatory network [8].

Salmonella has a second MarA-like protein called RamA, an AraC/XylS transcription activator synthesised from ramA [9]. ramA is a homologue of the E. coli marA gene, having $45 \%$ similarity [10]; while not present in E. coli, ramA is present in a wide range of Enterobacteriaceae. Like MarA in E. coli, RamA positively regulates $a c r A B$ expression in $S$. enterica $[11,12]$ by binding to the region upstream of $\operatorname{acr} A B$. RamR is the transcriptional repressor of $\operatorname{ram} R$ and $\operatorname{ram} A$, binding to the ramRA intergenic region $[13,14]$. We include the ramRA regulatory region in Salmonella's gene regulatory network (Fig. 1(C)) and omit the mar operon due to previous studies showing inactivation of marR and marA (as well as soxR, and soxS) did not affect the susceptibilities of a $S$. enterica serovar Typhimurium strain, whereas inactivating ramR resulted in a multidrug resistance phenotype [13]. This observation, along with other supporting studies, suggests the ramRA regulatory region primarily controls $A c r A B$ and efflux-mediated multidrug resistance in Salmonella $[11,15]$. The major roles of the mar operon and ramRA region in regulating acrAB expression, in E. coli and Salmonella respectively, supports the choice of these regulators in our coarse-grained GRNs (Fig. 11(B)-(C)). Additional regulation of $a c r A B$ is provided locally in both species by $\operatorname{acr} R[16,17]$. Its product, AcrR, is known to repress both its own synthesis and $a c r A B$ from a divergently transcribed region [16]. Under normal conditions, acrR is transcribed at a basal level, preventing over-expression of $\operatorname{acr} A B[16,18]$.

The expression of efflux pumps is inducible by the presence of environmental signals, or chemical compounds (such as antibiotics). The inducibility of efflux genes enables bacteria to adapt to a wide range of environmental conditions. Noxious substances or stressors can bind to transcription factors MarR, RamR and AcrR. In E. coli, derepression of the mar operon can occur through stress molecules reversibly binding to MarR, inhibiting its ability to bind to DNA and interfering with the repressor activity of MarR $[19,20]$. Derepression of mar, by the MarR inhibitor salicylate for example [21], promotes increased transcription of marRAB, in particular marA, and consequently $\operatorname{acr} A B[19,20]$. The activity of $A c r R$, which belongs to the TetR family of transcriptional repressors, has been observed to be induced by ligands such as rhodamine 6G, ethidium, and proflavin [22]. In Salmonella, substances increase acrAB expression through the increased expression of both ramA and ramR, by inhibiting the binding of RamR to the $P_{\text {ramA }}$ region $[14,23]$. Studies have shown that not all antibiotics significantly increase the expression of ramA [24], suggesting that not all efflux substrates are molecules that induce efflux expression. In our model we consider the effect of molecules that induce efflux expression and can be exported by the efflux pump, displayed through a repressive interaction from $a c r A B$ to a stress node $S$ in Supplementary Fig. $S 1$. 


\subsection{Additional Actors in Gene Regulatory Networks}

The regulatory models considered in this study focus on transcript level behaviour, excluding additional regulatory processes, such as Lon protease and CsrA, acting at the post-transcriptional or post-translational level to fine-tune the regulation of $a c r A B$ expression [25-27]. Additional layers of control from, for example, soxRS, rob and acrZ, are also known to be integrated into regulating AcrAB expression [28-31]. These regulators are thought to play a minor role in $a c r A B$ regulation, or efflux-related resistance, compared to the mar, ram and acrR regulators [11,13,18, 29, 32,33].

Feedback between efflux genes and transcriptional regulators has also been identified [24,34-36], however, full knowledge of their mechanism, including whether it is direct or indirect, is still incomplete; in the case where the relationship between the regulatory players is stated, but the potential interactions are still unknown, they are omitted from the GRNs. Further, bacterial efflux pump regulation is highly interlinked, with various efflux pumps previously shown to display compensatory expression when alternative efflux pump genes are up- or down-regulated. In Salmonella, for example, expression of $a c r D$ and acrF genes, encoding homologous RND pumps in enterobacteria, were significantly up-regulated in an acr $B$ knockout mutant [35-37]. Likewise, analogous behaviour is observed for acrB expression when $\triangle$ acrF mutants, or $\triangle a c r B \Delta a c r F$ double mutants are considered [35]. AcrAB-TolC is a clinically relevant multidrug resistance efflux pump within E. coli and Salmonella [38-40]. The key involvement of AcrAB in clinical resistance corroborates the focus of our study on the regulation of this efflux pump component. 


\subsection{Validating the Theoretical Model Through Independent Experimental Observa- tions}

Supplementary Table S1 contains multiple independent experimental observations captured by our theoretical model, and used to validate our mathematical approach. weights.

Table S1: Theoretical model reproduces realistic behaviour in E. coli and Salmonella. Each row in the table describes experimentally observed behaviour (with references) and theoretical results arising from our model that capture analogous behaviour. References supporting experimental observations are labelled according to the dependence of information contained within each reference that is applied to construct the gene regulatory networks. The reference markers show: $(\bullet)$ a dependent study where observations are used to flow into GRN construction, making them more difficult to disambiguate from model construction; ( • ) a study where the mechanism of the stated observation is unspecified, but other results are used in

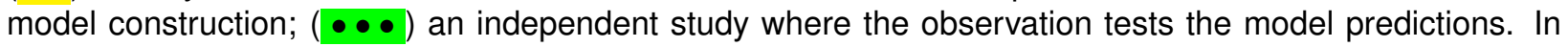
summary, references with more dots have a higher strength of independence, and are a better validation of the model.

\begin{tabular}{|c|c|c|}
\hline Experimental Observation & $\begin{array}{l}\text { Theoretical Observation from } \\
\text { Boolean Model }\end{array}$ & References \\
\hline $\begin{array}{l}\text { An isogenic, uninduced E. coli } \\
\text { population displays significant } \\
\text { cell-to-cell variability in marA } \\
\text { expression. }\end{array}$ & $\begin{array}{l}\text { In the absence of stress, the sim- } \\
\text { ulated behaviour of marA displays } \\
\text { significant cell-to-cell expression vari- } \\
\text { ability at the population and single- } \\
\text { cell level. At low, intermediate, and } \\
\text { high energy availability, marA ex- } \\
\text { pression level coefficient of varia- } \\
\text { tion (ELCV) is approximately } 1.0 \text { in } \\
\text { each energy sub-population (Fig. } 2 \\
\text { (ii)). The population-level observa- } \\
\text { tions predict a collective population } \\
\text { of low, intermediate, and high en- } \\
\text { ergy sub-populations of cells would } \\
\text { contain high cell-to-cell variability in } \\
\text { marA expression. A qualitative inves- } \\
\text { tigation of marA expression dynamics } \\
\text { in simulated single-cells further sup- } \\
\text { ports the heterogeneity observed at } \\
\text { the population-level (Supplementary } \\
\text { Fig. S6). }\end{array}$ & $\begin{array}{l}\bullet \bullet \text { [41]: Fig. } 1 \mathrm{C}, 2 \mathrm{~B} \text { and } \\
\text { Fig. } 1 \mathrm{C} \text { shows cell-to-cell } \\
\text { variability in marA expression } \\
\text { at different time points. } \\
\text { Fig. } 2 \mathrm{~B} \text { plots single-cell } \\
\text { readouts of the marA pro- } \\
\text { moter (at } t=0 \text { min). } \\
\text { Fig. } 4 \mathrm{C} \text { is a histogram } \\
\text { showing frequency (\%) of } \\
\text { cells with a given marA } \\
\text { expression level. }\end{array}$ \\
\hline
\end{tabular}




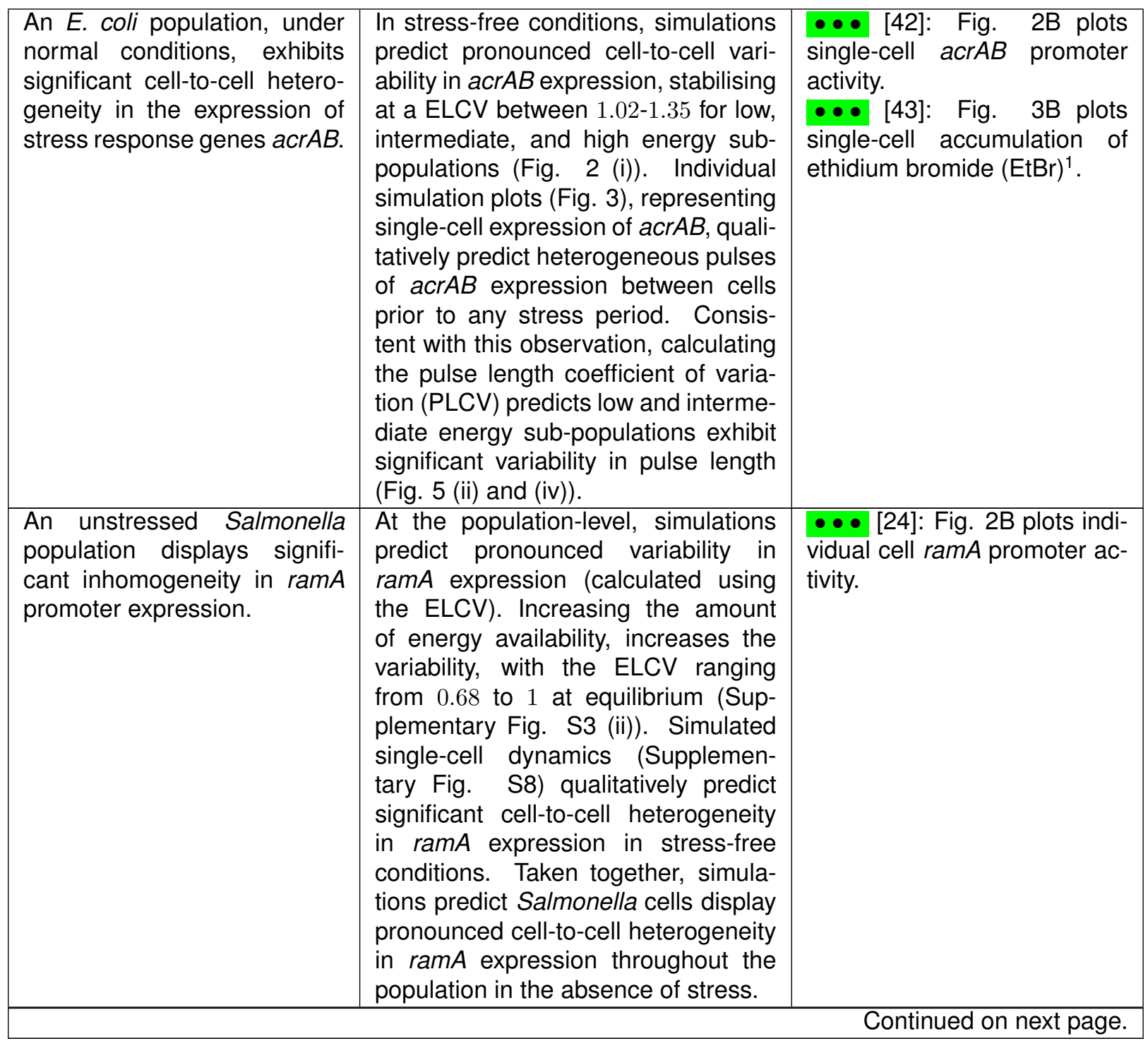

\footnotetext{
${ }^{1}$ This observation applies to a broader range of efflux genes, other than the one we consider, as well as cell membrane protein complexes involved in the uptake of small molecules (e.g. porins). However, our model considers the main efflux genes in E. coli, and it is highly probable the experiments correlate to the $a c r A B$ genes, not the minor players.
} 


\begin{tabular}{|c|c|c|}
\hline $\begin{array}{l}\text { Salmonella enterica serovar } \\
\text { Typhimurium, under normal } \\
\text { conditions, exhibits heteroge- } \\
\text { neous cell-to-cell efflux pump } \\
\text { expression in a population. }\end{array}$ & $\begin{array}{l}\text { Population-level simulations (Supple- } \\
\text { mentary Fig. S3 (i)) predict high } \\
\text { variability in acrAB expression within } \\
\text { low, intermediate and high energy } \\
\text { sub-populations (calculated through } \\
\text { the ELCV). The ELCV increases } \\
\text { with the amount of energy avail- } \\
\text { able, ranging from } 0.86 \text { to } 1.45 \text {, } \\
\text { suggesting significant heterogeneity } \\
\text { as energy increases. In agree- } \\
\text { ment, simulated Salmonella single- } \\
\text { cells qualitatively predict heteroge- } \\
\text { neous cell-to-cell acrAB expression } \\
\text { in low, intermediate, and high energy } \\
\text { sub-populations (Supplementary Fig. } \\
\text { S7). Taken together, this biologi- } \\
\text { cally suggests a Salmonella popula- } \\
\text { tion would contain considerable cell- } \\
\text { to-cell variability in acrAB expression } \\
\text { in the absence of stress. }\end{array}$ & $\begin{array}{l}\bullet \bullet\left[\left.44\right|^{2} \text { Fig. } 5 \mathrm{~A} \text { and } \mathrm{B}\right. \\
\text { plots EtBr accumulation in an } \\
\text { isogenic culture of } S \text {. enterica. }\end{array}$ \\
\hline $\begin{array}{l}\text { The marRAB operon can } \\
\text { be induced by numerous } \\
\text { unrelated stress compounds, } \\
\text { increasing transcriptional ac- } \\
\text { tivity of marRAB in wild-type } \\
\text { E. coli. } \\
\text { Data shows tetracy- } \\
\text { cline [3, 45, 46], chloram- } \\
\text { phenicol [45], sodium sal- } \\
\text { icylate [5, 47, 48], some } \\
\text { redox-cycling compounds [4] } \\
\text { and the uncoupling agent car- } \\
\text { bonyl cyanide m-chlorophenyl } \\
\text { hydrazone [4] all induce mar } \\
\text { transcript. }\end{array}$ & $\begin{array}{l}\text { The application of stress within } \\
\text { simulations triggers a response in } \\
\text { marRA, increasing the mean expres- } \\
\text { sion within low, intermediate and } \\
\text { high energy sub-populations (Fig. } \\
2 \text { (ii)). Simulated single-cell be- } \\
\text { haviour is consistent with population- } \\
\text { level predictions, qualitatively display- } \\
\text { ing an increase in transcriptional ac- } \\
\text { tivity of marRA when induced by a } \\
\text { stressor (Supplementary Fig. S6]. } \\
\text { The increase in marRA expression is } \\
\text { more pronounced as energy availabil- } \\
\text { ity and stress length increases (Sup- } \\
\text { plementary Fig. S6. }\end{array}$ & $\begin{array}{l}\bullet \text { [3]: Fig. 4, WT column. } \\
\bullet \text { [4]: Table 3, column } 3 . \\
\bullet \text { [5]: Fig. 6. } \\
\bullet \bullet \text { [45]: Fig. 5A lanes 1-3. } \\
\bullet \bullet \bullet \text { [46]: Table 1, row } 1 . \\
\bullet \bullet \text { [47]: Fig. } 2 \text { lanes } 1 \text { and } \\
2 . \\
\bullet \bullet \text { [48]: Fig. 2A and B, col- } \\
\text { umn 1. }\end{array}$ \\
\hline $\begin{array}{l}\text { Stress molecules, including } \\
\text { fatty acids, bile salts, ethanol } \\
\text { and } \mathrm{NaCl}[2,16] \text {, induce } E \text {. } \\
\text { coli acrAB expression, in- } \\
\text { creasing the transcript level. }\end{array}$ & $\begin{array}{l}\text { Population-level simulations predict } \\
\text { an induction in acrAB expression in } \\
\text { the presence of a simulated stressor, } \\
\text { for all energy levels (Fig. } 2 \text { (i)). Sim- } \\
\text { ulated single-cell stress responses } \\
\text { suggest the increase in transcrip- } \\
\text { tional activity is more pronounced } \\
\text { and more dynamic as energy avail- } \\
\text { ability and stress period increase. }\end{array}$ & $\begin{array}{l}\bullet \bullet[2]: \text { Fig. } 5 \text {. } \\
\bullet \bullet[16]: \text { Fig. } 3 \text { lanes } 1-2 \text { and } \\
\text { 5-6. }\end{array}$ \\
\hline
\end{tabular}

\footnotetext{
${ }^{2}$ Data is not acrAB specific, however, AcrAB is the major efflux pump in Salmonella, suggesting it is highly probable cell-to-cell differences in $\mathrm{EtBr}$ accumulation correlate to variability in acr $A B$ gene activity, not the minor players. To support this, Fig. 5B shows a strong similarity in EtBr accumulation for wild-type (WT) cells in the presence of an efflux pump inhibitor and $\triangle a c r A B$ cells.
} 


\begin{tabular}{|c|c|c|}
\hline $\begin{array}{l}\text { Transcription of acrR in- } \\
\text { creases under ethanol and } \\
\mathrm{NaCl} \text { stress conditions. }\end{array}$ & $\begin{array}{l}\text { In simulated stress conditions, the } \\
\text { behaviour of acrR, in both E. coli and } \\
\text { Salmonella, displays an induction of } \\
\text { expression at the population level. At } \\
\text { low, intermediate, and high energy } \\
\text { availability, mean acrR expression in- } \\
\text { creases (Fig. 2 (ii) and Supplemen- } \\
\text { tary Fig. S3 (ii)). The population- } \\
\text { level observations suggest a collec- } \\
\text { tive population of low, intermediate, } \\
\text { and high energy sub-populations of } \\
\text { cells would display an increase in } \\
\text { acrR expression when exposed to a } \\
\text { stressor. }\end{array}$ & $\begin{array}{l}\bullet[16] \text { : Fig. } 3 A \text {, compare } \\
\text { lanes } 3 \text { and } 7 \text { to lanes } 4 \text { and } \\
8 \text { respectively (this instance is } \\
\text { for WT E. coli cells). }\end{array}$ \\
\hline $\begin{array}{l}\text { Noxious substances increase } \\
\text { ramA, and subsequently } \\
\text { acrAB, expression in wild- } \\
\text { type Salmonella cells. } \\
\text { Data shows bile [11, 14], } \\
\text { indole [24, 49,50], select } \\
\text { biocides [24] and antibiotics } \\
\text { (including chloramphenicol, } \\
\text { ciprofloxacin, cloxacillin and } \\
\text { rifampicin) [24], induce ramA } \\
\text { and acrAB expression. }\end{array}$ & $\begin{array}{l}\text { Population-level behaviour in simu- } \\
\text { lated stress conditions predict an ini- } \\
\text { tiation of a stress response at each } \\
\text { energy level. This response generally } \\
\text { consists of an initial increase in tran- } \\
\text { scriptional activity of ramR and ramA } \\
\text { within each energy sub-population } \\
\text { (Supplementary Fig. S3 (ii) and (iv)). } \\
\text { The induced ramA expression posi- } \\
\text { tively modulates efflux pump genes } \\
\text { acrAB, increasing the mean expres- } \\
\text { sion of acrAB in each sub-population } \\
\text { (Supplementary Fig. S3 (i) and (iii)). } \\
\text { In agreement, simulated single-cell } \\
\text { dynamics qualitatively predict an in- } \\
\text { crease in ramA transcriptional activ- } \\
\text { ity (Supplementary Fig. S8), followed } \\
\text { by a transition to the active efflux ex- } \\
\text { pression state (Supplementary Fig. } \\
\text { S7) in the presence of stress, within } \\
\text { low, intermediate, and high energy } \\
\text { Salmonella single-cells. }\end{array}$ & $\begin{array}{l}\bullet \bullet \text { [11]: Figs. } 1 \mathrm{~A}, 2 \text { and } 3 . \\
\bullet[14]: \text { Fig. } 1 \mathrm{~A} . \\
\bullet \bullet \bullet \text { [24]: Fig. } 3 \text { A-C and } \\
\text { Supplementary Fig. S5. } \\
\bullet \bullet \bullet \text { [49]: Fig. } 1 \text { C-2 and Ta- } \\
\text { ble 2. } \\
\bullet \bullet \bullet \text { [50]: Fig. 1A and B. }\end{array}$ \\
\hline
\end{tabular}




\subsection{Salmonella and E. coli wiring diagram schematics}

Supplementary Fig. S1 shows the wiring diagram version of E. coli (Fig. 1(B)) and Salmonella (Fig. 1(C)) efflux pump gene regulatory networks, including the regulatory interactions illustrating inactivation of transcription factors by noxious substances, and the capacity of the assembled efflux pump (AcrAB-TolC) to remove stressors.

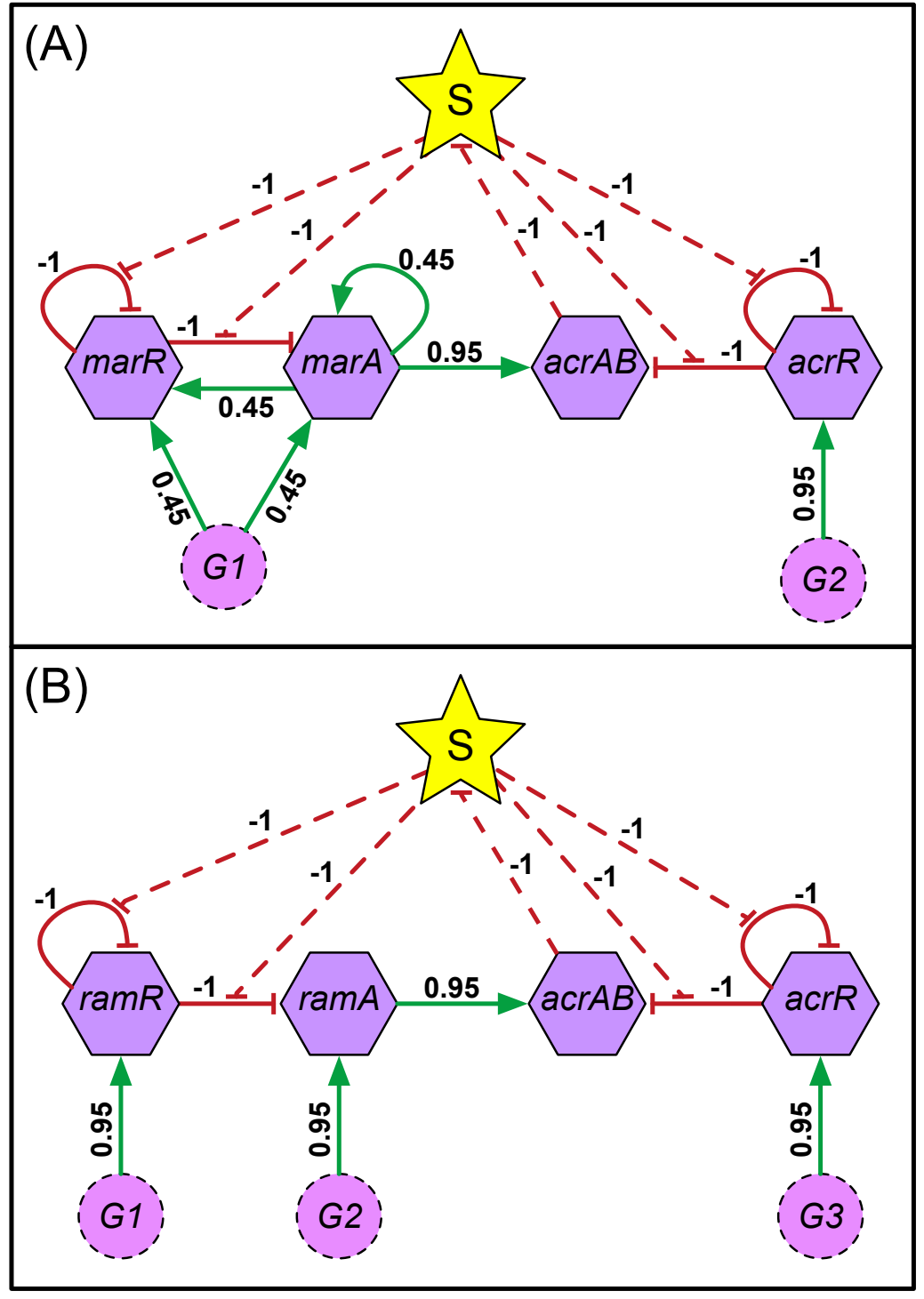

Figure S1: Schematic representation of network wiring diagrams. Wiring diagrams for (A) E. coli and (B) Salmonella efflux pump GRNs (Fig. 1(B) and (C), respectively). GRN interactions directly modulate network nodes in the wiring diagram depiction, and stress-related regulation (dashed edges to and from node $S$ ) modulates network regulatory edges; edge weights within the wiring diagram are derived from the literature, and enable each element in the network a chance to act. Each regulatory interaction is colour coded to display positive (green) or negative (red) operations. 


\subsection{Network dynamics from different initial conditions}

The first question we addressed with our model was whether the initial state of the network led to different long-term behaviour in E. coli and Salmonella cells in environments with and without a stress. We define the stressed and unstressed initial conditions (ICs) for our model by considering the active components in bacterial efflux mechanisms in an environment with and without a stressor (see Methods). We then simulated the evolution of each network over a set time period, starting either from an unstressed state or a stressed state applied for 1 or 25 timesteps (modelling different stress conditions). The stressor was applied by perturbing the network after reaching a population equilibrium, and not during the initial transient activity of the network.

We found that the ICs had no influence on the long-term behaviour of either network in simulated stressfree conditions - for example, compare Fig. 2 and Supplementary Fig. S2 before the short stress period in E. coli (similarly, compare the pre-stress behaviour in Supplementary Figs. S3 and S4 for Salmonella). When a stress is applied to a network, the response of the network and long-term dynamics are unaffected by the ICs. The analogous response to stress for both ICs is not surprising, as each network is perturbed once population equilibrium - which is the same for both ICs - is reached. Therefore, in the absence of energy availability, the choice of IC does not affect the long-term behaviour.

When allowing for energy variability within the model, identical results were observed (compare Fig. 2 to Supplementary Fig. S2 for E. coli), supporting the use of the unstressed initial condition within this study. 
(i)

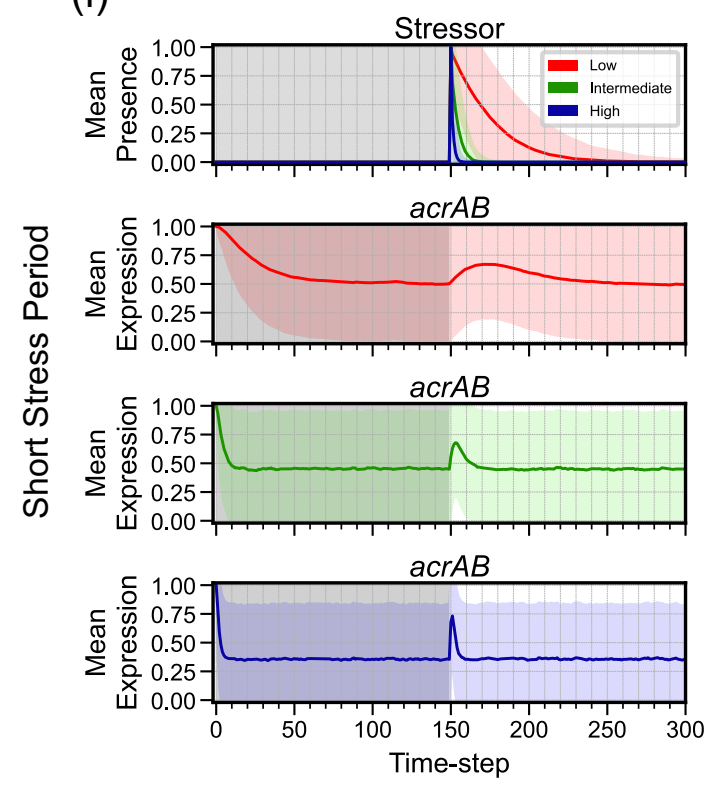

(iii)

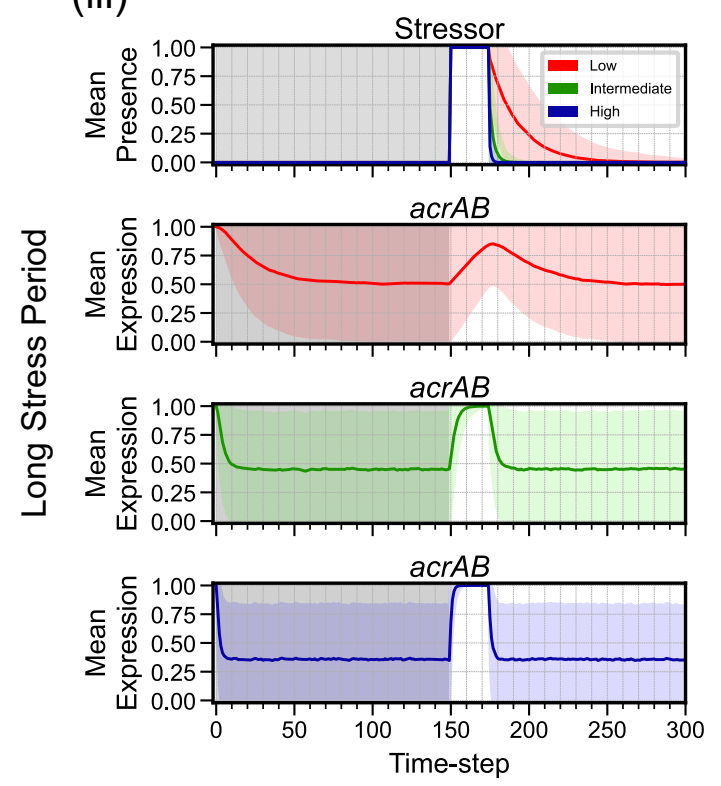

(ii)
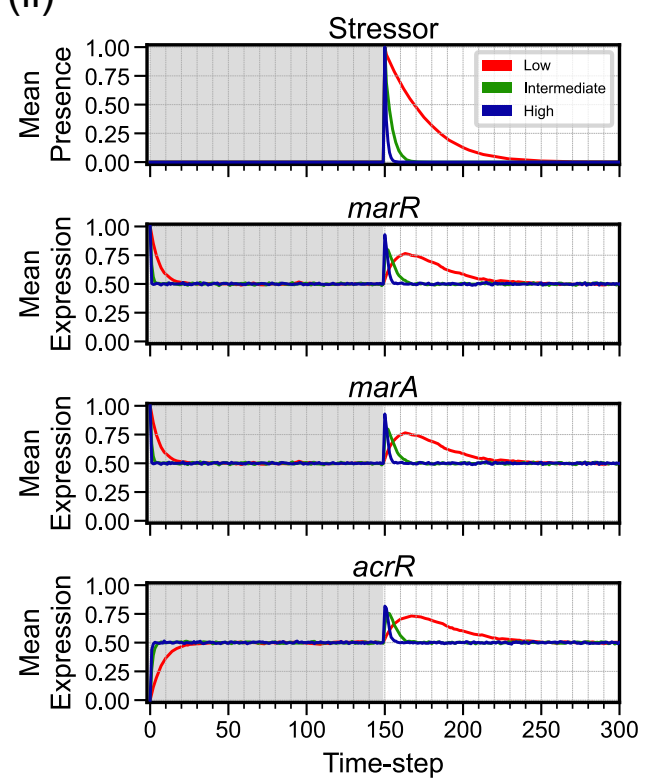

(iv)
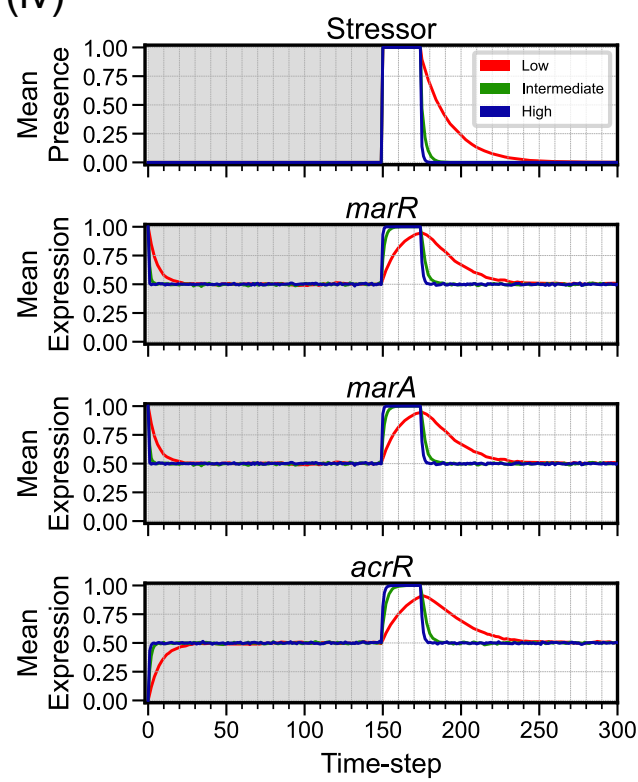

Figure S2: $E$. coli mean expression dynamics are equivalent from different initial conditions. Timeseries dynamics of mean expression (coloured line for low (red), intermediate (green) and high (blue) energy levels) for ((i), (iii)) efflux genes acrAB and ((ii), (iv)) remaining regulatory components in E. coli, from the stressed initial condition; all panels include stressor behaviour. Behaviour is shown when the network is exposed to a (i)-(ii) short $(t=150)$ or (iii)-(iv) long $(t=150-174)$ stress period. The initial time region of each regulatory component prior to stress application (grey shaded region) displays the complete stress-free behaviour. Note: Standard deviation (corresponding coloured shaded area) is included within efflux panels to represent the uncertainty, but the actual expression level cannot exceed the $[0,1]$ domain. 
(i)

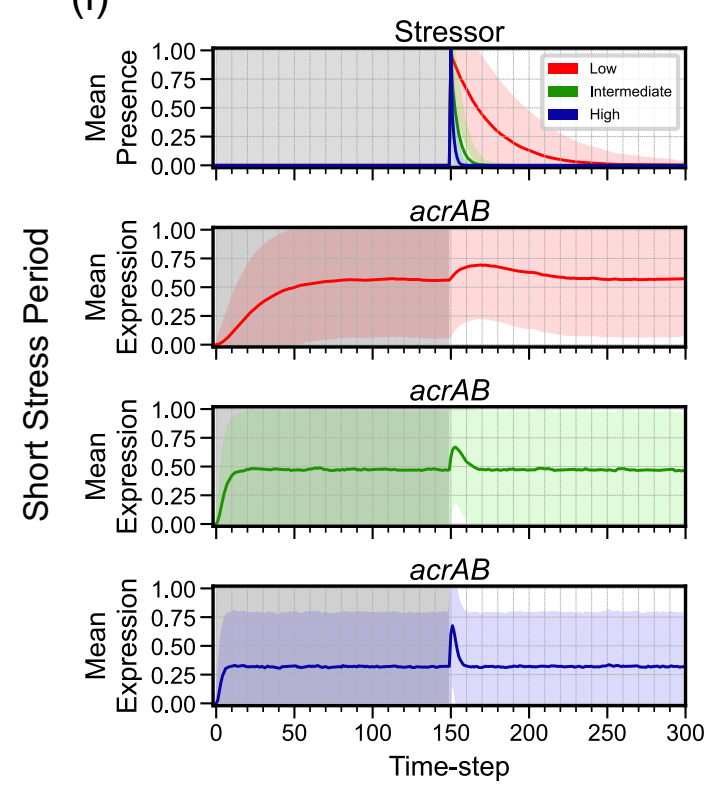

(iii)

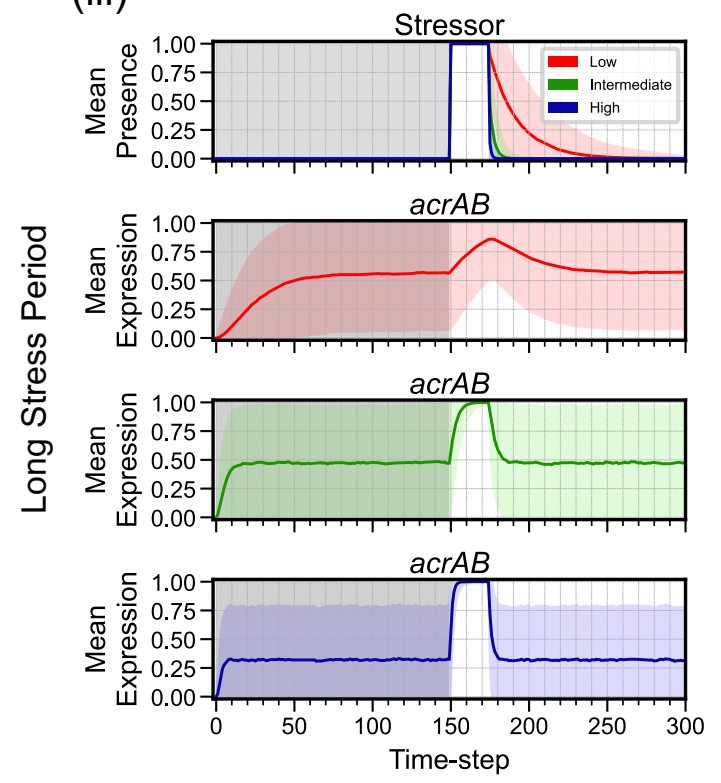

(ii)
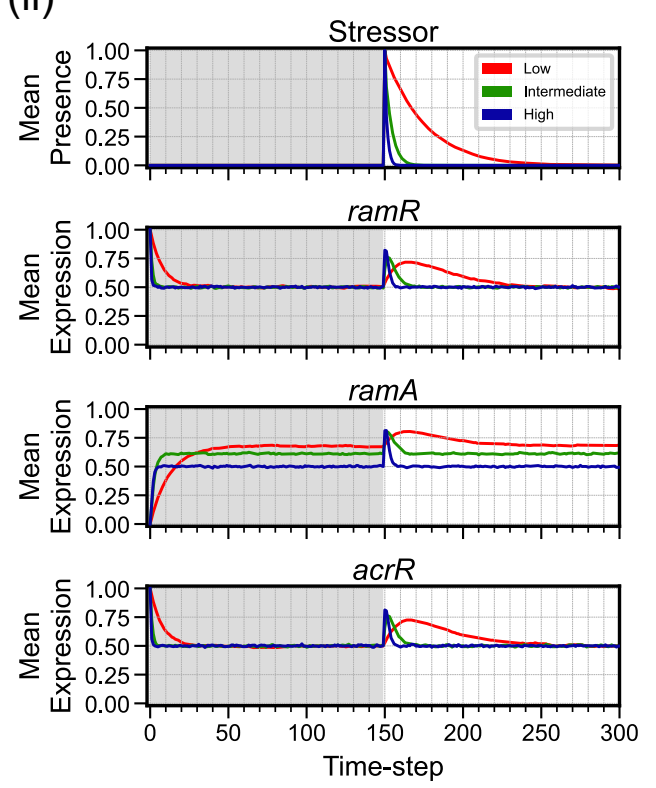

(iv)
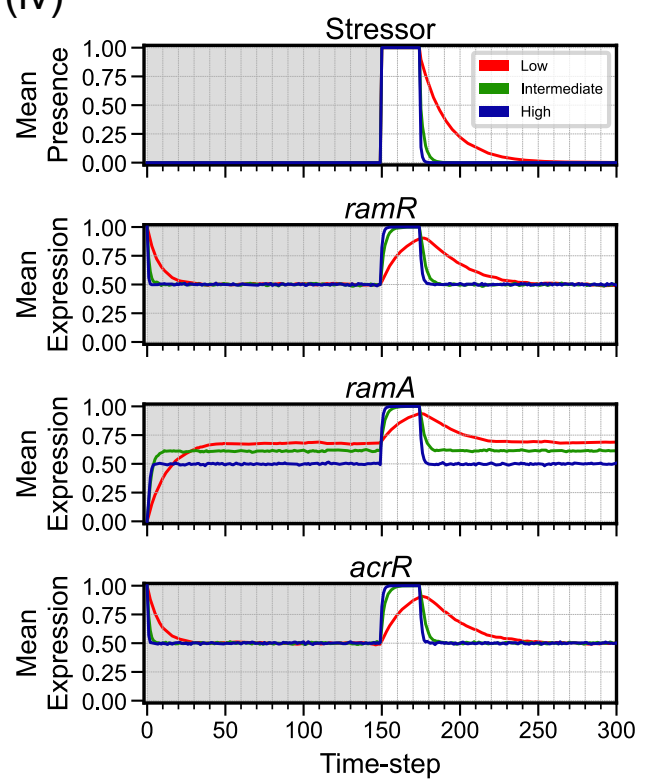

Figure S3: Mean expression dynamics for Salmonella from the unstressed initial condition in response to a short or long stressor duration. Time-series dynamics of mean expression (coloured line for low (red), intermediate (green) and high (blue) energy levels) for ((i), (iii)) efflux genes acrAB and ((ii), (iv)) remaining regulatory components in Salmonella, from the unstressed initial condition; all panels include stressor behaviour. Behaviour is shown when the network is exposed to a (i)-(ii) short $(t=150)$ or (iii)-(iv) long ( $t=150-174)$ stress period. The initial time region of each regulatory component prior to stress application (grey shaded region) displays the complete stress-free behaviour. Note: Standard deviation (corresponding coloured shaded area) is included within efflux panels to represent the uncertainty, but the actual expression level cannot exceed the $[0,1]$ domain. 
(i)

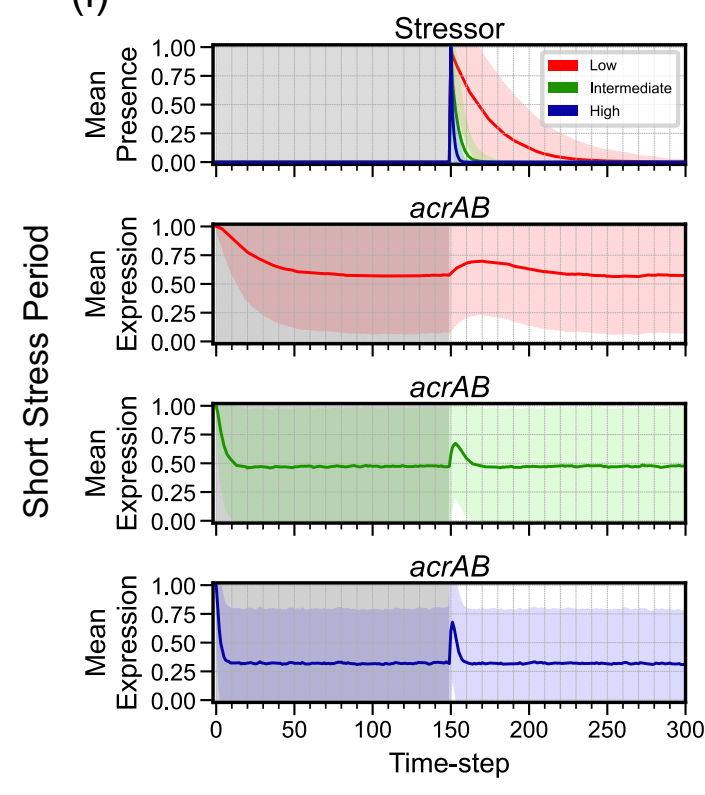

(iii)

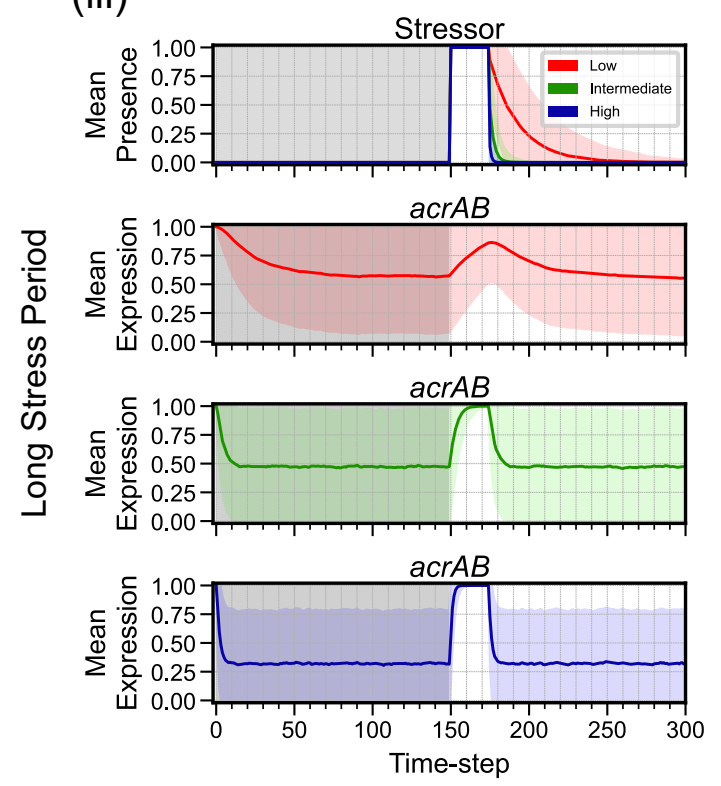

(ii)
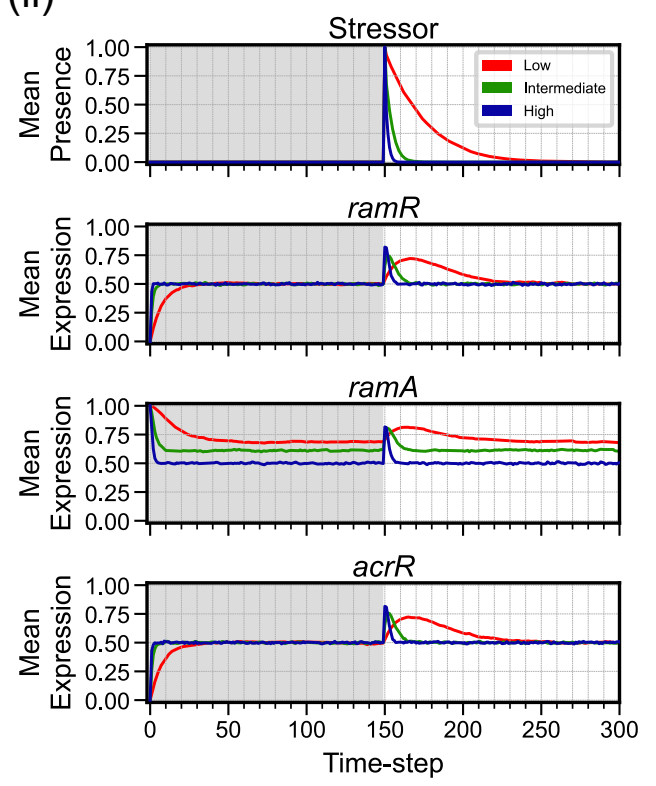

(iv)
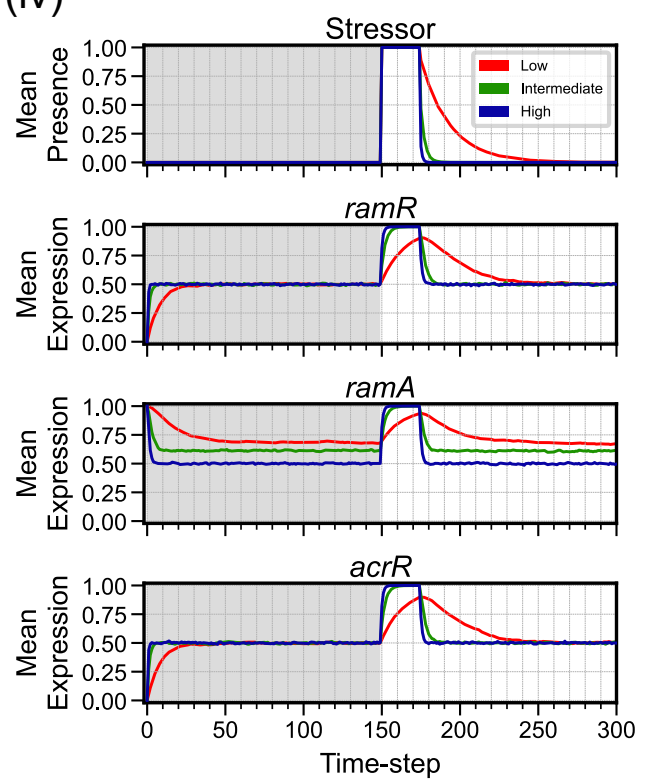

Figure S4: Salmonella mean expression dynamics are equivalent from different initial conditions. Time-series dynamics of mean expression (coloured line for low (red), intermediate (green) and high (blue) energy levels) for ((i), (iii)) efflux genes acrAB and ((ii), (iv)) remaining regulatory components in Salmonella, from the stressed initial condition; all panels include stressor behaviour. Behaviour is shown when the network is exposed to a (i)-(ii) short $(t=150)$ or (iii)-(iv) long $(t=150-174)$ stress period. The initial time region of each regulatory component prior to stress application (grey shaded region) displays the complete stress-free behaviour. Note: Standard deviation (corresponding coloured shaded area) is included within efflux panels to represent the uncertainty, but the actual expression level cannot exceed the $[0,1]$ domain. 
bioRxiv preprint doi: https://doi.org/10.1101/2021.12.13.472361; this version posted December 14,2021 . The copyright holder for this preprint (which was not certified by peer review) is the author/funder, who has granted bioRxiv a license to display the preprint in perpetuity. It is made available under aCC-BY 4.0 International license.

\subsection{Salmonella network transitions}

Supplementary Fig. S5 displays network state transitions for Salmonella GRN (Fig. 1(C)) using heatmaps, with and without stress.

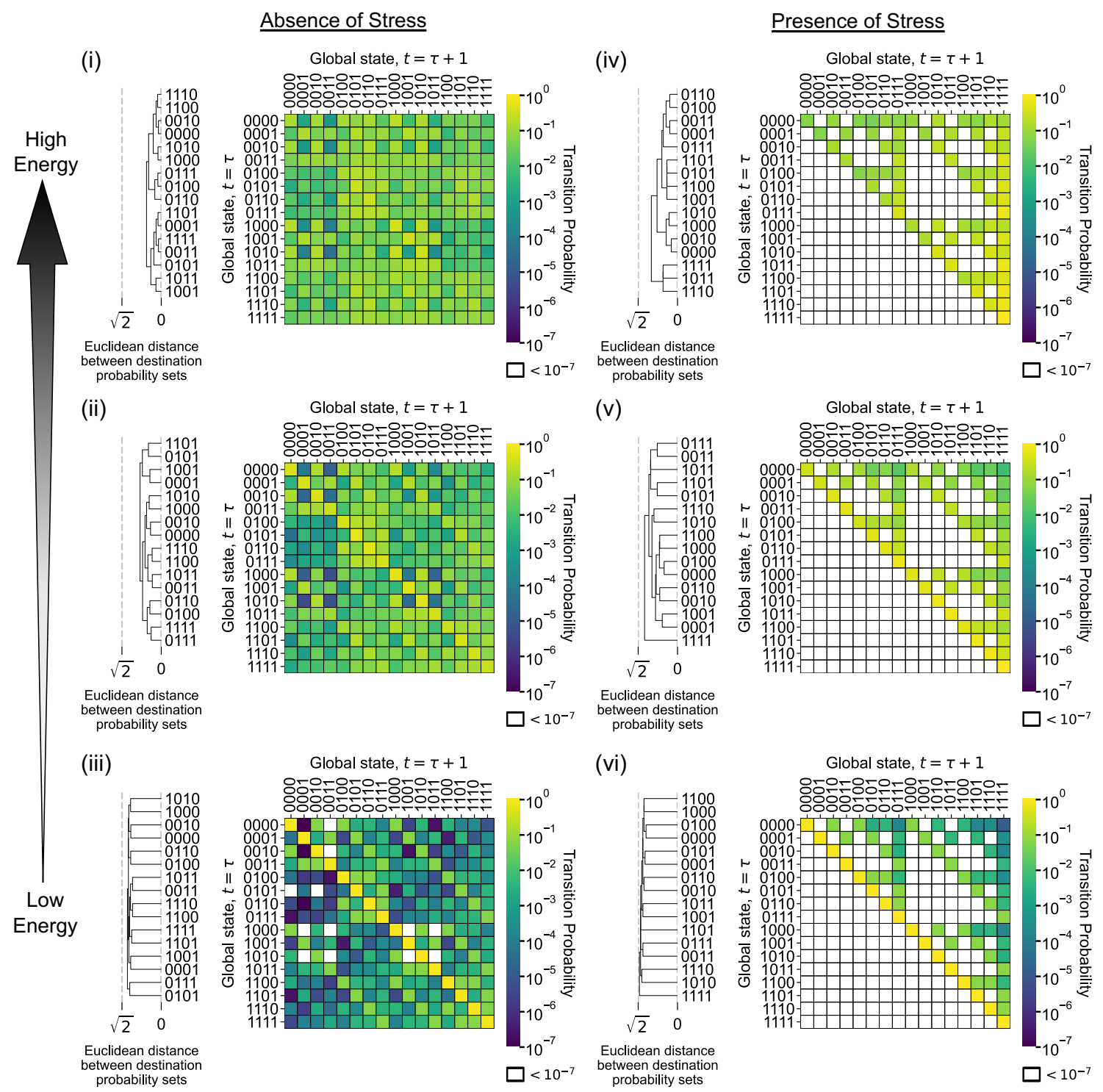

Figure S5: Salmonella displays analogous coarse-grained qualitative behaviour compared to E. coli. Heatmap plots showing Salmonella's transition matrix. Each row contains the transition probability distribution for each of $2^{M}$ possible global states, in the absence (i)-(iii) and presence (iv)-(vi) of a stressor, with increasing energy availability; $M$ here is the number of nodes in the initial GRN. Each matrix element is coloured to indicate the probability $p$ (using a logarithmic scale) that global state A transitions to global state $\mathrm{B}$ from timestep $t=\tau$ to $t=\tau+1$. Hierarchical clustering of global states is calculated using the destination possibilities $(t=\tau+1)$ for each $t=\tau$ global state, with clustering employing a Euclidean distance metric to determine the distances between each element. Global state binary vectors are displayed in the order [ramR, $\operatorname{ramA}, \operatorname{acr} R, \operatorname{acr} A B]$. 
bioRxiv preprint doi: https://doi.org/10.1101/2021.12.13.472361; this version posted December 14, 2021. The copyright holder for this preprint (which was not certified by peer review) is the author/funder, who has granted bioRxiv a license to display the preprint in perpetuity. It is made available under aCC-BY 4.0 International license.

\section{${ }_{735} 5.7$ Individual Cell Dynamics Display Significant Cell-to-Cell Heterogeneity}

${ }_{736}$ Supplementary Figs. S6 S8 display qualitative dynamics of simulated single-cell marRA expression in $E$. ${ }_{737}$ coli, and acrAB and ramA expression in Salmonella, respectively. 
bioRxiv preprint doi: https://doi.org/10.1101/2021.12.13.472361; this version posted December 14, 2021. The copyright holder for this preprint (which was not certified by peer review) is the author/funder, who has granted bioRxiv a license to display the preprint in perpetuity. It is made available under aCC-BY 4.0 International license.
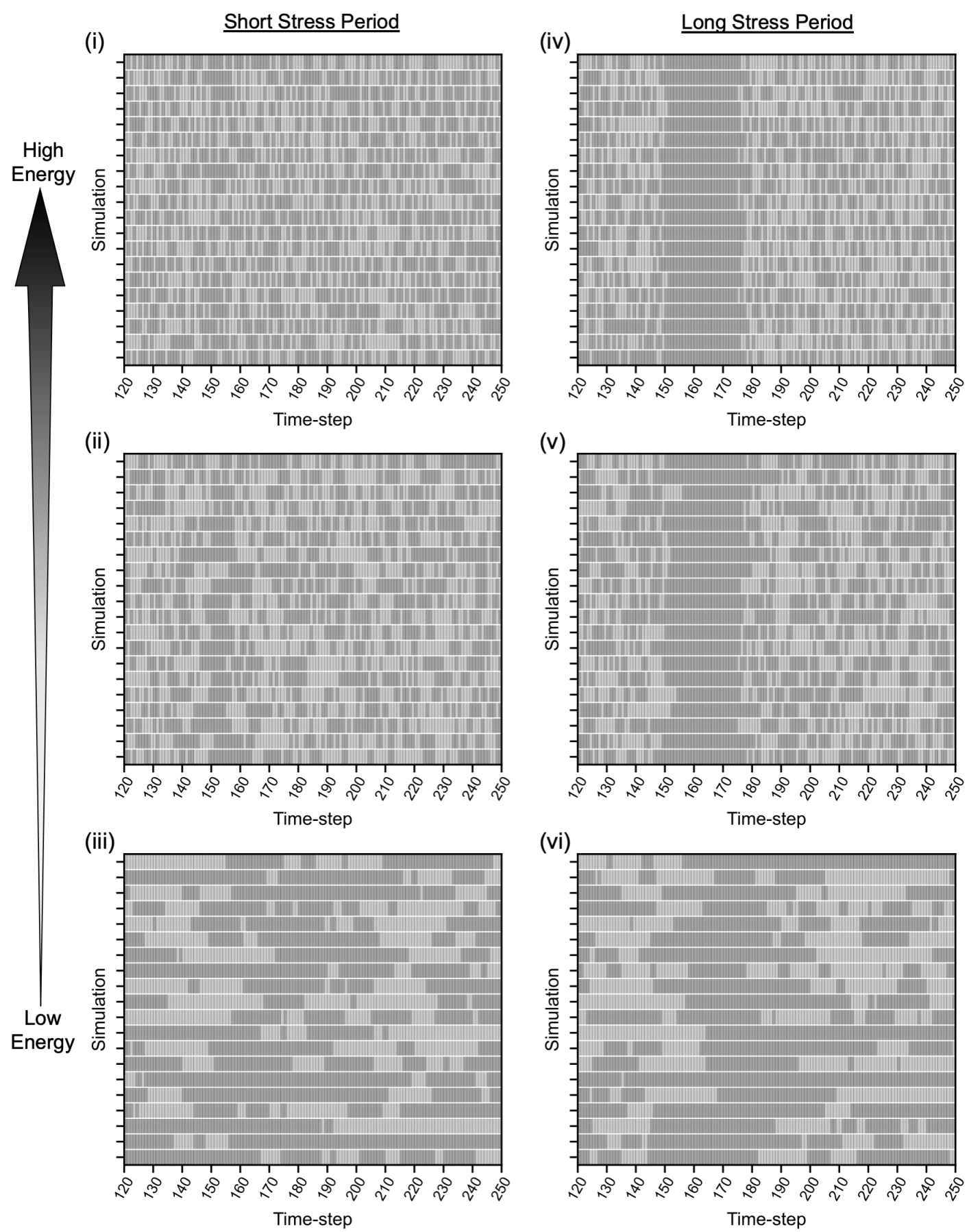

Figure S6: E. coli displays significant heterogeneity in marRA gene expression in the absence of stress. Qualitative plots displaying marRA "on"/"off" (dark/light grey) dynamics for 20 randomly selected individual E. coli simulations, when exposed to a (i)-(iii) short or (iv)-(vi) long stressor period. Panels display marRA dynamics between timesteps $t=120-250$ at low, intermediate, and high energy availability. The short stressor period is a single timestep pulse activated at $t=150$ and the long stressor period is activated at $t=150$ until $t=174$. 
bioRxiv preprint doi: https://doi.org/10.1101/2021.12 13.472361; this version posted December 14, 2021. The copyright holder for this preprint (which was not certified by peer review) is the author/funder, who has granted bioRxiv a license to display the preprint in perpetuity. It is made available under aCC-BY 4.0 International license.
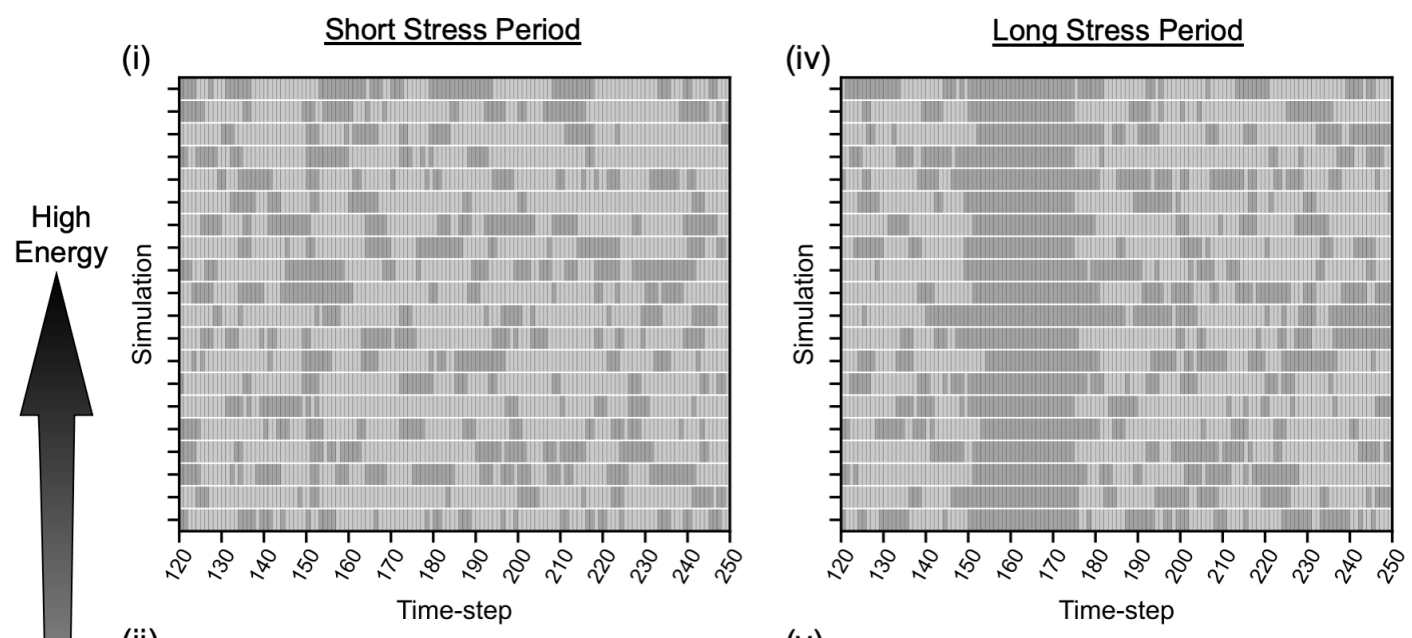

(ii)

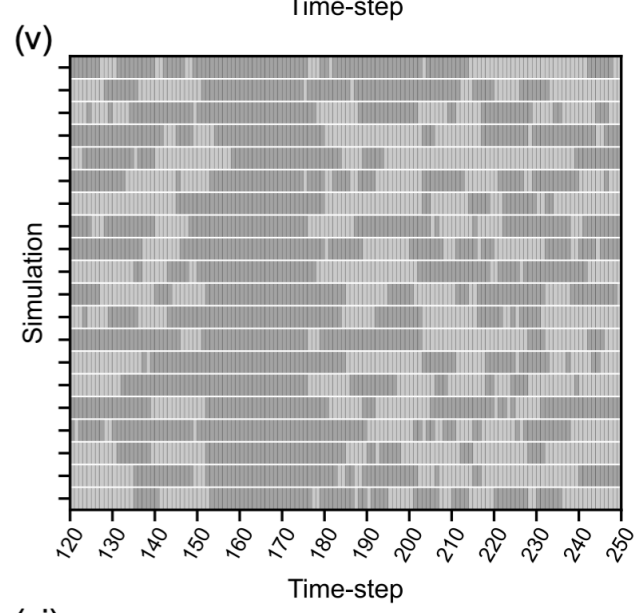

(iii)
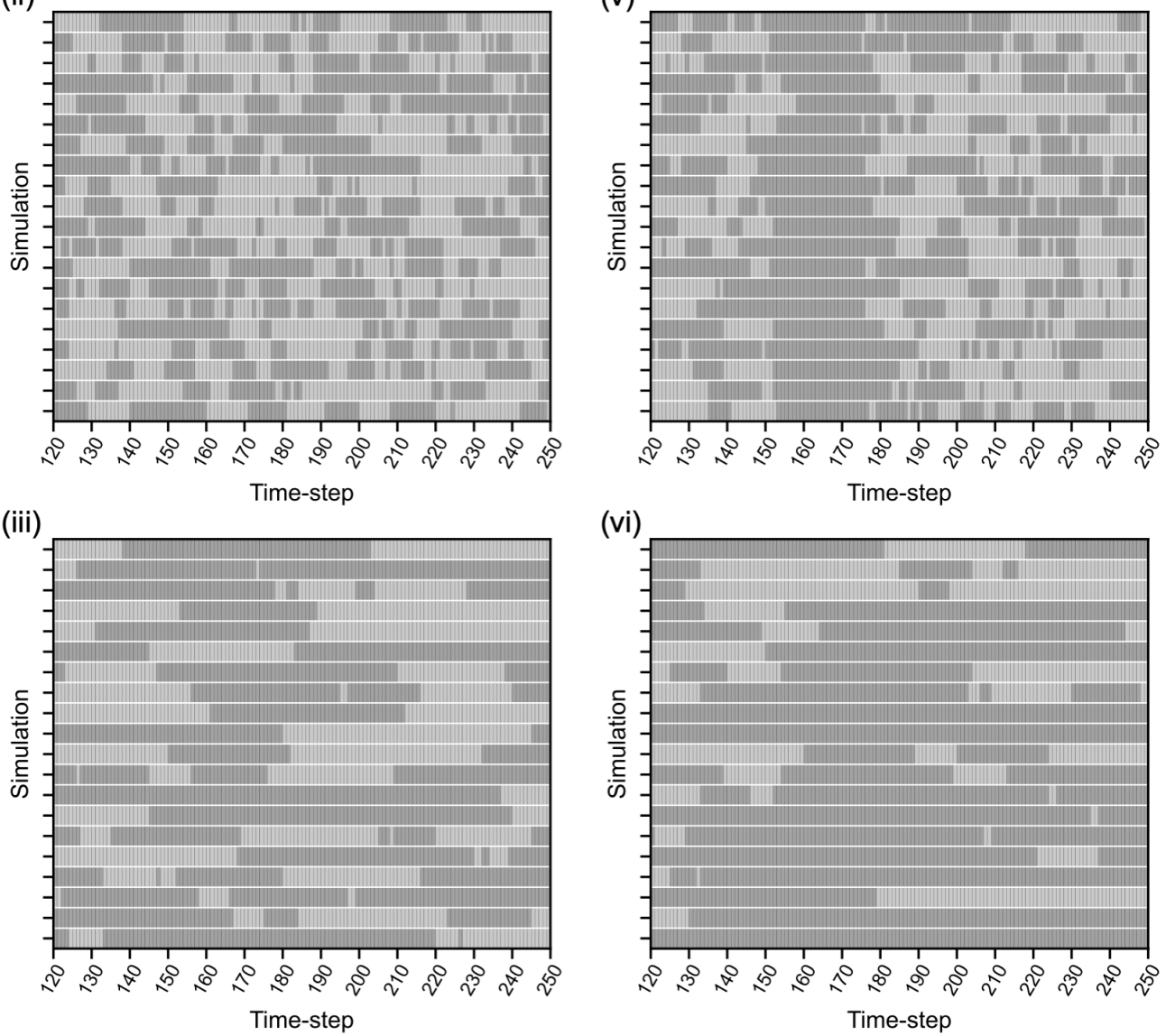

Figure S7: Simulations predict Salmonella expresses acrAB in heterogeneous pulses in the absence of stress. Qualitative plots displaying acrAB "on"/"off" (dark/light grey) dynamics for 20 randomly selected individual Salmonella simulations, when exposed to a (i)-(iii) short or (iv)-(vi) long stressor period. Panels display acrAB dynamics between timesteps $t=120-250$ at low, intermediate, and high energy availability. The short stressor period is a single timestep pulse activated at $t=150$ and the long stressor period is activated at $t=150$ until $t=174$. 
bioRxiv preprint doi: https://doi.org/10.1101/2021.12.13.472361; this version posted December 14, 2021. The copyright holder for this preprint (which was not certified by peer review) is the author/funder, who has granted bioRxiv a license to display the preprint in perpetuity. It is made available under aCC-BY 4.0 International license.
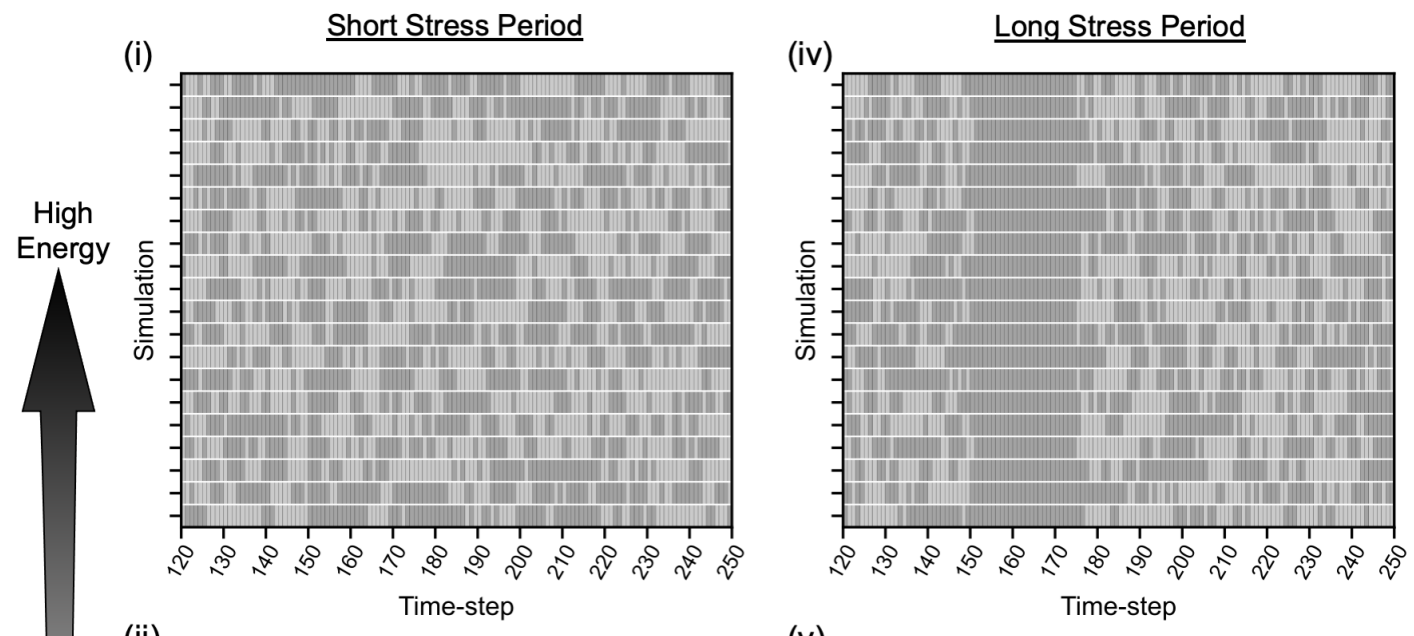

(ii)

Time-step

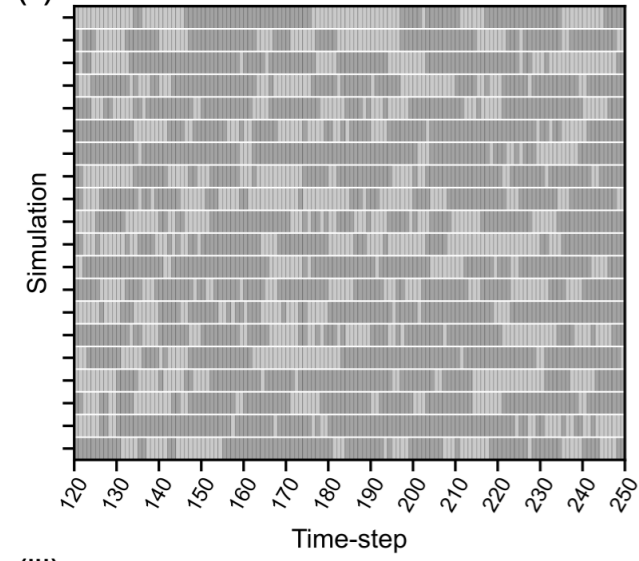

(iii)
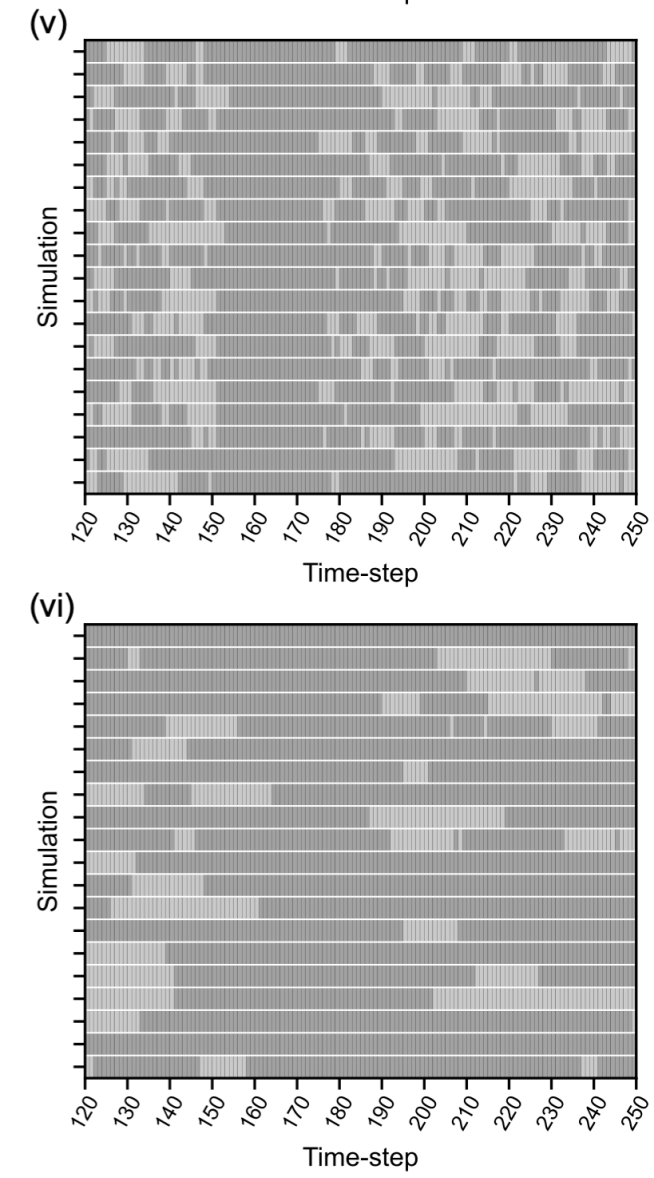

Figure S8: Salmonella displays significant heterogeneity in ramA gene expression in the absence of stress. Qualitative plots displaying ramA "on"/"off" (dark/light grey) dynamics for 20 randomly selected individual Salmonella simulations, when exposed to a (i)-(iii) short or (iv)-(vi) long stressor period. Panels display ramA dynamics between timesteps $t=120-250$ at low, intermediate, and high energy availability. The short stressor period is a single timestep pulse activated at $t=150$ and the long stressor period is activated at $t=150$ until $t=174$. 


\section{References}

[1] Seth P Cohen, H Hächler, and SB Levy. Genetic and functional analysis of the multiple antibiotic resistance (mar) locus in escherichia coli. Journal of bacteriology, 175(5):1484-1492, 1993.

[2] Dzwokai Ma et al. Genes acra and acrb encode a stress-induced efflux system of escherichia coli. Molecular microbiology, 16(1):45-55, 1995.

[3] RR Ariza, SP Cohen, NANDITA Bachhawat, SB Levy, and Bruce Demple. Repressor mutations in the marrab operon that activate oxidative stress genes and multiple antibiotic resistance in escherichia coli. Journal of bacteriology, 176(1):143-148, 1994.

[4] Asuncion S Seoane and Stuart B Levy. Characterization of marr, the repressor of the multiple antibiotic resistance (mar) operon in escherichia coli. Journal of bacteriology, 177(12):3414-3419, 1995.

[5] Robert G Martin, Kam-Wing Jair, RE Wolf, and Judah L Rosner. Autoactivation of the marrab multiple antibiotic resistance operon by the mara transcriptional activator in escherichia coli. Journal of bacteriology, 178(8):2216-2223, 1996.

[6] Laura M McMurry, AM George, and SB Levy. Active efflux of chloramphenicol in susceptible escherichia coli strains and in multiple-antibiotic-resistant (mar) mutants. Antimicrobial agents and chemotherapy, 38(3):542-546, 1994.

[7] Teresa M Barbosa and Stuart B Levy. Differential expression of over 60 chromosomal genes in escherichia coli by constitutive expression of mara. Journal of bacteriology, 182(12):3467-3474, 2000.

[8] Laura Vinué, Laura M McMurry, and Stuart B Levy. The 216-bp marb gene of the marrab operon in escherichia coli encodes a periplasmic protein which reduces the transcription rate of mara. FEMS microbiology letters, 345(1):49-55, 2013.

[9] Tahar van der Straaten, Riny Janssen, Dik J Mevius, and Jaap T van Dissel. Salmonella gene rma (rama) and multiple-drug-resistant salmonella enterica serovar typhimurium. Antimicrobial agents and chemotherapy, 48(6):2292-2294, 2004.

[10] Valérie Duval and Ida M Lister. Mara, soxs and rob of escherichia coli-global regulators of multidrug resistance, virulence and stress response. International journal of biotechnology for wellness industries, 2(3):101, 2013.

[11] Eiji Nikaido, Akihito Yamaguchi, and Kunihiko Nishino. Acrab multidrug efflux pump regulation in salmonella enterica serovar typhimurium by rama in response to environmental signals. Journal of Biological Chemistry, 283(35):24245-24253, 2008.

[12] Vito Ricci and Laura JV Piddock. Ciprofloxacin selects for multidrug resistance in salmonella enterica serovar typhimurium mediated by at least two different pathways. Journal of antimicrobial chemotherapy, 63(5):909-916, 2009.

[13] Yousef M Abouzeed, Sylvie Baucheron, and Axel Cloeckaert. ramr mutations involved in effluxmediated multidrug resistance in salmonella enterica serovar typhimurium. Antimicrobial agents and chemotherapy, 52(7):2428-2434, 2008.

[14] Sylvie Baucheron et al. Bile-mediated activation of the acrab and tolc multidrug efflux genes occurs mainly through transcriptional derepression of rama in salmonella enterica serovar typhimurium. Journal of Antimicrobial Chemotherapy, 69(9):2400-2406, 2014.

[15] Masaru Usui, Hidetaka Nagai, Mototaka Hiki, Yutaka Tamura, and Tetsuo Asai. Effect of antimicrobial exposure on acrab expression in salmonella enterica subspecies enterica serovar choleraesuis. Frontiers in microbiology, 4:53, 2013. 
[16] Dzwokai Ma et al. The local repressor acrr plays a modulating role in the regulation of acrab genes of escherichia coli by global stress signals. Molecular microbiology, 19(1):101-112, 1996.

[17] Anne Olliver, Michel Vallé, Elisabeth Chaslus-Dancla, and Axel Cloeckaert. Role of an acrr mutation in multidrug resistance of in vitro-selected fluoroquinolone-resistant mutants of salmonella enterica serovar typhimurium. FEMS microbiology letters, 238(1):267-272, 2004.

[18] Mark A Webber, Ashraf Talukder, and Laura JV Piddock. Contribution of mutation at amino acid 45 of acrr to acrb expression and ciprofloxacin resistance in clinical and veterinary escherichia coli isolates. Antimicrobial agents and chemotherapy, 49(10):4390-4392, 2005.

[19] Michael N Alekshun and Stuart B Levy. Alteration of the repressor activity of marr, the negative regulator of the escherichia coli marrab locus, by multiple chemicals in vitro. Journal of bacteriology, 181(15):4669-4672, 1999.

[20] Robert G Martin and Judah L Rosner. Binding of purified multiple antibiotic-resistance repressor protein (marr) to mar operator sequences. Proceedings of the National Academy of Sciences, 92(12):5456-5460, 1995.

[21] Niranjana Sri Sundaramoorthy et al. Restoring colistin sensitivity in colistin-resistant e. coli: combinatorial use of marr inhibitor with efflux pump inhibitor. Scientific Reports, 9(1):1-13, 2019.

[22] Chih-Chia Su, Denae J Rutherford, and W Yu Edward. Characterization of the multidrug efflux regulator acrr from escherichia coli. Biochemical and biophysical research communications, 361(1):85-90, 2007.

[23] Suguru Yamasaki et al. The crystal structure of multidrug-resistance regulator ramr with multiple drugs. Nature communications, 4(1):1-7, 2013.

[24] AJ Lawler, V Ricci, SJW Busby, and LJV Piddock. Genetic inactivation of acrab or inhibition of efflux induces expression of rama. Journal of Antimicrobial Chemotherapy, 68(7):1551-1557, 2013.

[25] Kevin L Griffith, Ishita M Shah, and Richard E. Wolf Jr. Proteolytic degradation of escherichia coli transcription activators soxs and mara as the mechanism for reversing the induction of the superoxide (soxrs) and multiple antibiotic resistance (mar) regulons. Molecular microbiology, 51(6):1801-1816, 2004.

[26] Vito Ricci, Jessica MA Blair, and Laura JV Piddock. Rama, which controls expression of the mdr efflux pump acrab-tolc, is regulated by the lon protease. Journal of Antimicrobial Chemotherapy, 69(3):643650, 2014.

[27] Vito Ricci et al. Csra maximizes expression of the acrab multidrug resistance transporter. Nucleic acids research, 45(22):12798-12807, 2017.

[28] Rafael R Ariza, Ziyi Li, Niels Ringstad, and Bruce Demple. Activation of multiple antibiotic resistance and binding of stress-inducible promoters by escherichia coli rob protein. Journal of bacteriology, 177(7):1655-1661, 1995.

[29] Errett C Hobbs, Xuefeng Yin, Brian J Paul, Jillian L Astarita, and Gisela Storz. Conserved small protein associates with the multidrug efflux pump acrb and differentially affects antibiotic resistance. Proceedings of the National Academy of Sciences, 109(41):16696-16701, 2012.

[30] Robert G Martin and Judah L Rosner. Genomics of the mara/soxs/rob regulon of escherichia coli: identification of directly activated promoters by application of molecular genetics and informatics to microarray data. Molecular microbiology, 44(6):1611-1624, 2002. 
[31] Paul F Miller, Laura F Gambino, Mark C Sulavik, and Stephen J Gracheck. Genetic relationship between soxrs and mar loci in promoting multiple antibiotic resistance in escherichia coli. Antimicrobial agents and chemotherapy, 38(8):1773-1779, 1994.

[32] Marjon HJ Bennik, Pablo J Pomposiello, Derek F Thorne, and Bruce Demple. Defining a rob regulon in escherichia coliby using transposon mutagenesis. Journal of bacteriology, 182(13):3794-3801, 2000.

[33] Laura JV Piddock. Clinically relevant chromosomally encoded multidrug resistance efflux pumps in bacteria. Clinical microbiology reviews, 19(2):382-402, 2006.

[34] Andrew M Bailey, lan T Paulsen, and Laura JV Piddock. Rama confers multidrug resistance in salmonella enterica via increased expression of acrb, which is inhibited by chlorpromazine. Antimicrobial agents and chemotherapy, 52(10):3604-3611, 2008.

[35] Deborah J Eaves, Vito Ricci, and Laura JV Piddock. Expression of acrb, acrf, acrd, mara, and soxs in salmonella enterica serovar typhimurium: role in multiple antibiotic resistance. Antimicrobial agents and chemotherapy, 48(4):1145-1150, 2004.

[36] Xuan Wang-Kan et al. Lack of acrb efflux function confers loss of virulence on salmonella enterica serovar typhimurium. MBio, 8(4), 2017.

[37] Vito Ricci et al. Ciprofloxacin-resistant salmonella enterica serovar typhimurium strains are difficult to select in the absence of acrb and tolc. Antimicrobial Agents and Chemotherapy, 50(1):38-42, 2006.

[38] Martin J Everett, Yu Fang Jin, Vito Ricci, and LJ Piddock. Contributions of individual mechanisms to fluoroquinolone resistance in 36 escherichia coli strains isolated from humans and animals. Antimicrobial agents and chemotherapy, 40(10):2380-2386, 1996.

[39] Sylvie Baucheron et al. Acrab-tolc directs efflux-mediated multidrug resistance in salmonella enterica serovar typhimurium dt104. Antimicrobial agents and chemotherapy, 48(10):3729-3735, 2004.

[40] Laura JV Piddock et al. Evidence for an efflux pump mediating multiple antibiotic resistance in salmonella enterica serovar typhimurium. Antimicrobial Agents and Chemotherapy, 44(11):31183121, 2000.

[41] Imane El Meouche, Yik Siu, and Mary J Dunlop. Stochastic expression of a multiple antibiotic resistance activator confers transient resistance in single cells. Scientific reports, 6(1):1-9, 2016.

[42] Imane El Meouche and Mary J Dunlop. Heterogeneity in efflux pump expression predisposes antibioticresistant cells to mutation. Science, 362(6415):686-690, 2018.

[43] Laura Paixão et al. Fluorometric determination of ethidium bromide efflux kinetics in escherichia coli. Journal of biological engineering, 3(1):18, 2009.

[44] María Antonia Sánchez-Romero and Josep Casadesús. Contribution of phenotypic heterogeneity to adaptive antibiotic resistance. Proceedings of the National Academy of Sciences, 111(1):355-360, 2014.

[45] H Hächler, Seth P Cohen, and Stuart B Levy. mara, a regulated locus which controls expression of chromosomal multiple antibiotic resistance in escherichia coli. Journal of bacteriology, 173(17):55325538, 1991.

[46] Mark C Sulavik, Laura F Gambino, and Paul F Miller. The marr repressor of the multiple antibiotic resistance (mar) operon in escherichia coli: prototypic member of a family of bacterial regulatory proteins involved in sensing phenolic compounds. Molecular Medicine, 1(4):436-446, 1995. 
[47] Seth P Cohen, Stuart B Levy, John Foulds, and Judah L Rosner. Salicylate induction of antibiotic resistance in escherichia coli: activation of the mar operon and a mar-independent pathway. Journal of bacteriology, 175(24):7856-7862, 1993.

[48] WV Kern, M Oethinger, AS Jellen-Ritter, and SB Levy. Non-target gene mutations in the development of fluoroquinolone resistance in escherichia coli. Antimicrobial Agents and Chemotherapy, 44(4):814$820,2000$.

[49] Eiji Nikaido et al. Effects of indole on drug resistance and virulence of salmonella enterica serovar typhimurium revealed by genome-wide analyses. Gut pathogens, 4(1):1-13, 2012.

[50] Eiji Nikaido, Ikue Shirosaka, Akihito Yamaguchi, and Kunihiko Nishino. Regulation of the acrab multidrug efflux pump in salmonella enterica serovar typhimurium in response to indole and paraquat. Microbiology, 157(3):648-655, 2011. 